\title{
Mechanisms of Proton Conductance in Polymer Electrolyte Membranes
}

\author{
M. Eikerling, ${ }^{\dagger, *}$ A. A. Kornyshev, ${ }^{*, \dagger}$ A. M. Kuznetsov, ${ }^{\S}$ J. Ulstrup, ${ }^{\|}$and S. Walbran ${ }^{\dagger}$ \\ Institute for Materials and Processes in Energy Systems, Research Center "Jülich" GmbH, \\ D-52425 Jülich, Germany, The A. N. Frumkin Institute for Electrochemistry, Russian Academy of Sciences, \\ 117071 Moscow, Russia, and Department of Chemistry, The Technical University of Denmark, \\ DK-2800 Lyngby, Denmark
}

Received: September 5, 2000

\begin{abstract}
We provide a phenomenological description of proton conductance in polymer electrolyte membranes, based on contemporary views of proton transfer processes in condensed media and a model for heterogeneous polymer electrolyte membrane structure. The description combines the proton transfer events in a single pore with the total pore-network performance and, thereby, relates structural and kinetic characteristics of the membrane. The theory addresses specific experimentally studied issues such as the effect of the density of proton localization sites (equivalent weight) of the membrane material and the water content of the pores. The effect of the average distance between the sulfonate groups, which changes during membrane swelling, is analyzed in particular, and the factors which determine the temperature dependence of the macroscopic membrane conductance are disclosed. Numerical estimates of the specific membrane conductivity obtained from the theory agree very well with typical experimental data, thereby confirming the appropriateness of the theoretical concepts. Moreover, the versatility of the models offers a useful and transparent frame for combining the analysis of both experimental data and the results of molecular dynamics simulations.
\end{abstract}

\section{Introduction}

The levels of research and development of new proton conductors as well as those currently available have increased dramatically over the past decade. ${ }^{1-6}$ This activity has been driven by the booming demand for fuel cell technology, particularly for vehicles and portable applications. ${ }^{7}$ These impose rather strict requirements on proton conductors, such as stable performance in the temperature range between 80 and $200{ }^{\circ} \mathrm{C}$ with a conductivity not lower than $0.1 \mathrm{~S} \mathrm{~cm}^{-2}$, chemical, mechanical, and thermal stability, and impermeability to gases, methanol, and charge carriers other than protons.

Some of these demands are met by the best currently available polymer electrolyte membranes such as Nafion, Aciplex, Flemion, and Dow membranes. These materials are essentially strong polymer acids. Exposed to water, they dissociate upon hydration into the immobile ionomer (anionic) groups residing on side chains of the polymer and free mobile protons in the aqueous solution. Thus, the free protons move through the hydrogen-bonded network of water molecules inside the polymer.

Compared to solid state proton conductors, the major advantage of aqueous polymer electrolyte conductors is the high conductivity of $0.01-0.1 \mathrm{~S} \mathrm{~cm}^{-2}$ available at moderate temperatures. This is due to the high concentration of protons in water-filled pores and the high proton mobility. The mobility is facilitated by a "collective" character of the proton transfer,

* To whom correspondence should be addressed. E-mail: a.kornyshev@ fz-juelich.de.

† Research Center "Jülich" GmbH

$\doteqdot$ Present address: Materials Science Division, Los Alamos National Laboratory, P.O. Box 1663, MS D429, Los Alamos, NM 87545.

$\S$ Russian Academy of Sciences.

" The Technical University of Denmark. which is induced by configuration fluctuations of donor and acceptor water molecules; this mechanism is discussed further below and is the basis for the model of proton conduction addressed in this paper. The main reason for the facile proton transport in aqueous systems is, however, simple. No matter how many "excess" protons there are in the system, all of them become indistinguishable in the "sea" of the background protons of water. There are thus no "fixed" and "free" protons; they are interchangeable. A proton, mobile a moment ago, takes a rest to become a part of a water molecule. The privilege to run is then relayed to another proton, which was a part of this molecule or of other molecules clustered with the first one. It is the relay character typical of aqueous hydrogen-bonded systems which makes proton transport fast.

While providing an environment for facile long-range proton transfer, aqueous-based membranes are also fraught with several disadvantages. One is the permeability to methanol, ${ }^{8}$ which makes it hard to use them in direct methanol fuel cells. They are also, obviously, unstable at elevated temperatures $(\gtrsim 100$ ${ }^{\circ} \mathrm{C}$ ), where electrocatalysis could otherwise run fast enough to consume all methanol at the anode so that the problem of methanol permeation would simply not arise. A third shortcoming is the electroosmotic effect: the transport of water induced by the classical migration of hydrated $\mathrm{H}_{3} \mathrm{O}^{+}$ions. This leads to dehydration of the anodic side of the membrane, and thus limits the current. ${ }^{9}$ Last, but not least, is the prohibitive aspect of high cost for the best-performing polymer electrolyte membranes, such as Nafion and its derivatives.

Progress in "dry" solid state proton conductors that offer high conductance without the listed deficiencies is, however, far from outclassing aqueous polymer electrolyte membranes in hydrogen energy technology. Theoretical and experimental efforts toward better understanding of membrane operation are therefore very 
much needed. This is especially true because such efforts are likely to shed new light on structural and chemical modifications which could improve the membrane performance.

The structure of the polymer electrolyte is very complex, and there is still no ultimate view of its detailed spatial organization. There is also no accepted picture of transport mechanisms. There are thus several ways to approach theoretically the structure and function of polymer electrolyte membranes. One way is to undertake molecular dynamic simulation studies, based on interatomic potentials $;^{10}$ in the context of polymer electrolyte membranes, such attempts are still in their infancy. Another way is to investigate idealized "physical" models, such as random or correlated network models, to compare rigorous solutions with experimental data, and in the case of success to explore them in the context of new predictions. There are, finally, ways based on phenomenological theories. ${ }^{11}$ Possibly having less predictive power, they could, however, provide transparent frameworks for the interpretation of computer simulations, experimental data, and model calculations.

Among the features of the membrane functions, which are first to be explained, are the proton conductivity dependence on temperature, equivalent weight (the number of grams of dry polymer per mole of anionic groups, in the following abbreviated as "EW"), and water uptake. Each of these observable correlations undoubtedly reflects several physical effects. These cannot necessarily be separated, but, framed by analytical theory, they can be illuminated and qualitatively assessed.

With such views, we provide in this report a semiphenomenological, theoretical description of proton conduction in polymer electrolyte membranes. We focus on membranes of the Nafion family, but the approach may be applied to a wider range of membranes. The description rests on models which are necessary but drastic simplifications of real membrane architecture and performance. In a different sense, the description, although crude, is comprehensive, as it addresses in principle all membrane components and available conduction features. Furthermore, it can be straightforwardly augmented or updated, as warranted by new structural or functional data, or simulation results.

The description covers the following three equally important elements of membrane proton conduction. (1) Proton transfer between localized ionic states or water molecules is considered as a fundamental chemical rate process. ${ }^{12}$ This part rests on the contemporary theory of proton transfer in condensed media, but the chemical identity of the donor and acceptor sites and the single-pore environment are crucial and specific to the membrane system. (2) In the next step, the proton transfer rate constant is converted to the proton conductance in a singlepore confinement. The key issue here is the calculation of the proton distribution function in the pore. In combination with the rate constant, it gives the single-pore conductivity. In the models, this also involves a crucial distinction between the proton conduction in the bulk of the pore and along the inner pore surface. The resulting single-pore conductivity yields interesting observations regarding the effects of temperature, pore dimensions (swelling), and density of proton localization sites (equivalent weight) on the proton conductivity. (3) The third part is the construction of the total pore network. This part involves a statistical approach to both fluctuations in the dimensions of individual pores and statistical combination of their conductances into the performance of the whole network. ${ }^{13,14}$ This part bridges the single-pore proton transfer events and the observable macroscopic membrane proton conductivity.
Equipped with such a theory, we discuss the available experimental data on membrane conductance and make some conclusions about the optimal membrane architecture.

\section{Mechanisms of Proton Transport in Water}

The motion of excess protons in water as a free proton, as a proton dressed in a polaron cloud, as a classical $\mathrm{H}_{3} \mathrm{O}^{+}$ion, or by mechanisms rooted in the Grotthus mechanism of structural diffusion is treated in a voluminous literature. ${ }^{15,16} \mathrm{New}$ gates toward detailed understanding of proton conduction at the molecular level appear to have been opened by recent ab initio quantum chemical and classical molecular dynamics simulations. ${ }^{15,17-19}$ The views emerging from these studies are also the basis for the investigation in this report, but we first discuss shortly a few general notions related to the chemical identity of the proton-conducting species in aqueous solutions and the nature of the fundamental physical event of proton transfer between dynamic proton carriers in aqueous solutions.

Due to the high rate of proton exchange in the hydrogenbonded system, the excess proton is accommodated equally among the other water protons. There is therefore no free excess proton in water. Three main options were discussed in the literature. ${ }^{20,21}$ First, the excess proton can be a part of an $\mathrm{H}_{3} \mathrm{O}^{+}$ ion, in which all three protons are equivalent. Second, it can reside in a Zundel ${ }^{20} \mathrm{H}_{5} \mathrm{O}_{2}{ }^{+}$cation complex with a proton between two water molecules, binding them in a cluster. Third, it can be present as an Eigen ${ }^{21} \mathrm{H}_{9} \mathrm{O}_{4}{ }^{+}$cluster, which consists of $\mathrm{H}_{3} \mathrm{O}^{+}$and three strongly bound $\mathrm{H}_{2} \mathrm{O}$ molecules, each attached to one of the $\mathrm{H}_{3} \mathrm{O}^{+}$protons. One can, of course, imagine other intermediate structural arrangements; the overall weight of the $\mathrm{H}_{5} \mathrm{O}_{2}{ }^{+}$and $\mathrm{H}_{9} \mathrm{O}_{4}{ }^{+}$clusters seems to be, however, dominant. In any case, all the clusters are short-lived in water, and their life and disappearance are crucial parts of the proton transport dynamics. The generic structural diffusion pathways of the three species are as follows.

A. The "Simplistic" Picture. In this view, the excess proton hops from the $\mathrm{H}_{3} \mathrm{O}^{+}$donor site to any neighboring water acceptor molecule, leaving a neutral water molecule behind. The proton hop is preceded by suitable configurational polarization fluctuations in water. The fluctuations appear as if water were polarized by the neighboring acceptor molecule, with the excess proton bound to the same extent as if it resided on the donor molecule. Such fluctuations determine the transition state. For the proton transfer (in the following abbreviated as PT) to occur, the electronic interaction between the donor and acceptor which triggers the transfer must, moreover, be strong enough.

The latter is only possible when the donor and acceptor acquire the proper coordination, resembling an $\mathrm{H}_{5} \mathrm{O}_{2}{ }^{+}$cluster. At the distance between the two oxygen atoms close to those of the equilibrium ground state of the $\mathrm{H}_{5} \mathrm{O}_{2}{ }^{+}$cluster in the gas phase $(2.4 \AA)$, the interaction between the $\mathrm{H}_{3} \mathrm{O}^{+}$and $\mathrm{H}_{2} \mathrm{O}$ moieties is so strong that there is no barrier to proton motion along the $\mathrm{O}-\mathrm{H}-\mathrm{O}$ coordinate and the proton is delocalized between the two water molecules. ${ }^{16,17}$ The equilibrium $\mathrm{O}-\mathrm{O}$ distance may be slightly longer in the condensed phase, still leaving a very small barrier, and a shallow double well may emerge.

The proton is displaced from the shallow double well by donor and acceptor molecular fluctuations out of the $\mathrm{H}_{5} \mathrm{O}_{2}{ }^{+}$ configuration. This makes the donor molecule " $\mathrm{H}_{2} \mathrm{O}$-looking" and the acceptor molecule " $\mathrm{H}_{3} \mathrm{O}^{+}$-looking". The $\mathrm{H}_{5} \mathrm{O}_{2}{ }^{+}$cluster is, thus, destroyed by such a fluctuation and the $\mathrm{H}_{3} \mathrm{O}^{+}$generated at a new site, displacing the excess charge over roughly the diameter of a water molecule. Overall, this is a collective 
mechanism for propagation of the "proton dislocation", in which many particles are involved, rather than consecutive singleproton hopping. The pathway for proton translocation is along any of the three protons of the initial $\mathrm{H}_{3} \mathrm{O}^{+}$ion, and the efficiency of such proton exchange exceeds by several-fold that of the classical diffusion of $\mathrm{H}_{3} \mathrm{O}^{+}$ion. ${ }^{22,23}$

B. Zundel's and Eigen's Picture. Comprehensive infrared spectral data have framed a view within which the $\mathrm{H}_{3} \mathrm{O}^{+}$ion is a less stable moelcular entity than the $\mathrm{H}_{5} \mathrm{O}_{2}{ }^{+}$complex. $\mathrm{H}_{5} \mathrm{O}_{2}{ }^{+}$ would then be the basic state of the proton, and the $\mathrm{H}_{5} \mathrm{O}_{2}{ }^{+}$ structural defect would move together with its charge in water. Again, this does not involve classical defect diffusion as a whole, but structural diffusion based on PT dominates and gives the high proton transport mobility. A water molecule, next to such a cluster, fluctuates into a configuration where it forms a new $\mathrm{H}_{5} \mathrm{O}_{2}{ }^{+}$together with the nearest molecule of the original $\mathrm{H}_{5} \mathrm{O}_{2}{ }^{+}$. This also requires orientational adjustment and deformation of both adjacent and remote water molecules in the original $\mathrm{H}_{5} \mathrm{O}_{2}{ }^{+}$ cluster. Subsequent fluctuations move remote water molecules out of the initial $\mathrm{H}_{5} \mathrm{O}_{2}{ }^{+}$configuration, shifting the charge by one water molecule. This motion may proceed along any of the four external protons of the $\mathrm{H}_{5} \mathrm{O}_{2}{ }^{+}$cluster.

Fluctuational preorganization followed by virtually barrierless PT carries over to mechanisms based on the $\mathrm{H}_{9} \mathrm{O}_{4}{ }^{+}$cluster. This is followed by decoupling of one of the peripheral molecules in the cluster, shifting the center of the cluster in the direction of the incoming acceptor water molecule. The process runs along one of the six peripheral protons.

Common to the three simplistic views is the fact that $\mathrm{H}^{+-}$ $n \mathrm{H}_{2} \mathrm{O}$ clusters self-assemble spontaneously up to a certain size. If a cluster were disrupted by the configurational fluctuations of the same molecule the fluctuational motion of which has created the cluster, there would be no charge transfer. If, however, the disruption comes from one of those molecules, which were a part of the initial cluster, the charge is shifted. In the class of models discussed above, the activation free energy of cluster motion is of the same order of magnitude as that for breaking a hydrogen bond.

Recent Kohn-Sham density functional simulations for molecular wires ${ }^{24}$ have shown that $\mathrm{H}_{3} \mathrm{O}^{+}$and $\mathrm{H}_{5} \mathrm{O}_{2}{ }^{+}$clusters are the dominant defects in water. Ab initio calculations in multimolecular water drops ${ }^{17}$ demonstrated the presence of all types of clusters in more or less equal amounts. The particular cluster size may be important for the proton conductivity of water in a confined geometry. Squeezing water inside a pore or embedding charged side chains may also change the distribution balance in the contribution of clusters of different size, since their condensed state energies are similar. In other words, the spatial constraints on the pathways via clusters of different size may affect the proton mobility. In our further construction, the size of the clusters will only affect certain parameters of the phenomenological theory.

\section{Proton Conductivity and Mobility in a Nafion Pore}

To a certain extent, concepts and models of proton conductivity in bulk water carry over to proton conductivity in Nafion and other polymer electrolyte membranes. A water-saturated, proton-conducting polymer electrolyte membrane behaves like an acid. Swollen in water, the $\mathrm{SO}_{3} \mathrm{H}$ groups of its side chains dissociate into $\mathrm{SO}_{3}{ }^{-}$anions and $\mathrm{H}^{+}$cations. The $\mathrm{SO}_{3}{ }^{-}$groups remain attached to the pore walls, whereas the protons are donated to water.

The proton mobility in the central region of a water-filled pore is expected to be close to the mobility in bulk water. ${ }^{25,26}$
However, near the surface it may be very different. In the bulk, all proton transfer takes place between symmetrical states, so while there is environmental nuclear reorganization associated with the transfer, in the absence of macroscopic driving forces the reaction Gibbs free energy or driving force is zero. At the surface, proton transfer cannot proceed directly between $\mathrm{SO}_{3}{ }^{-}$ groups because the average distance between these groups is far too large $(\sim 7-12 \AA) .{ }^{27} \mathrm{PT}$ is, therefore, invariably to intermediate sites different from the $\mathrm{SO}_{3}{ }^{-}$groups. These sites can hardly be any chemical species other than water, and in principle, surface proton conductivity involves an additional driving force contribution to the activation Gibbs free energy due to this asymmetry.

The precise state of the intermediate protonated water is not known, but the following considerations are an approach toward characterization. First of all, $\mathrm{SO}_{3} \mathrm{H}$ is a strong acid. Although dissociation involves an additional driving force contribution, this is not likely to be energetically a large quantity. Second, $\mathrm{PT}$ from $\mathrm{SO}_{3} \mathrm{H}$ to water in a direction perpendicular to the pore walls is thermodynamically unfavorable, at least for narrow pores, due to the cooperative electrostatic attraction of the proton both from the $\mathrm{SO}_{3}{ }^{-}$donor group and from several nearby $\mathrm{SO}_{3}{ }^{-}$ groups. PT along the pore wall toward a neighboring $\mathrm{SO}_{3}{ }^{-}$group is here much more feasible. Third, the state of the intermediate $\mathrm{H}_{2} \mathrm{O}$ and $\mathrm{H}_{3} \mathrm{O}^{+}$is then likely to be engaged in the hydrogen bond network, broadly similar to the hydrogen bond network in which $\mathrm{H}_{3} \mathrm{O}^{+}, \mathrm{H}_{5} \mathrm{O}_{2}{ }^{+}$, and $\mathrm{H}_{9} \mathrm{O}_{4}{ }^{+}$are engaged in bulk water. An $\mathrm{SO}_{3}{ }^{-}$interspacing of 7-8 $\mathrm{A}$ accommodates a single $\mathrm{H}_{3} \mathrm{O}^{+}$ in a configuration suitable for facile consecutive PT steps subject only to minor environmental configurational fluctuations and preorganization.

\section{Parametrization of the Proton Mobility in a Pore}

A. Bulk Transition Probability and Mobility. Common to the PT event that triggers structural diffusion in bulk water and at the pore surface is a strong interaction between the proton donor and acceptor. Ab initio electronic structure calculations and quantum path integral calculations, thus, concur with an extremely shallow or even vanishing barrier, once the transition configuration, preorganized for PT, is prepared. Proton tunneling is, therefore, only indirectly reflected in the PT transition probability in determining the residual barrier height for proton transfer. This is equivalent with the totally adiabatic limit known from molecular proton transfer theory. ${ }^{12}$ The transition probability in this limit takes the form

$$
W_{\mathrm{b}}=\frac{\omega_{\mathrm{b}}}{2 \pi} \exp \left(-\frac{\epsilon_{\mathrm{b}}+\frac{E_{\mathrm{b}}}{4}}{\theta}\right)
$$

Hereafter, the subscript b refers to the parameters of the bulk transfer. $E_{\mathrm{b}}$ is the reorganization Gibbs free energy of all the modes that contribute to the Franck-Condon factor. These are local translational, librational, and hydrogen bond deformational motions, but also bulk collective inertial polarization modes. $\epsilon_{\mathrm{b}}$ is a term which parametrizes the energy needed for the configurational preorganization of the solvated PT complex before the proton can move from the initial to the final state on the adiabatic potential energy surface. $\omega_{\mathrm{b}}$ is the effective frequency of all the environmental modes, ${ }^{28}$ and $\theta\left(=k_{\mathrm{B}} T\right)$ is the thermal energy, $k_{\mathrm{B}}$ being Boltzmann's constant and $T$ the temperature. Equation 1 represents a broad, parametrized PT rate constant form. It can be extended as warranted by straightforward interfacing with contemporary proton transfer theory, in particular as follows. 
(1) All environmental nuclear modes are regarded as linear and "classical", i.e., low-frequency. High-frequency nuclear tunneling effects, e.g., in the librational models $\left(\approx 800 \mathrm{~cm}^{-1}\right)$, anharmonicity, and mode mixing between the reactant and product states can, however, be incorporated as warranted.

(2) Equation 1 interpolates a variety of different specific reaction pathways, such as those based on the simplistic views and the more precise composite pathway rooted in the MarxParinello scheme and similar schemes.

The proton mobility can be estimated as ${ }^{28}$

$$
\mu_{\mathrm{b}}=\frac{k_{\mathrm{b}} v_{\mathrm{b}}}{2} \frac{e a_{\mathrm{b}}^{2} W_{\mathrm{b}}}{\theta}
$$

Here, $a_{\mathrm{b}}$ is the effective distance of the net charge transfer; this quantity is on the order of the "effective" diameter of a water molecule $(\sim 3 \AA$ ), but can be larger for cooperative two-proton transfer in larger clusters $(6-9 \AA)$. The geometrical factor $k_{\mathrm{b}}$ $\approx 1 / 3$ for "homogeneous and isotropic" bulk conductivity. $v_{\mathrm{b}}$ is the coordination number of the transfer. It would have a value of 3 for the simplistic mechanism, 4 for the Zundel mechanism, and 6 for the Eigen mechanism of structural diffusion. As none of these models uniquely determines proton transfer pathways, $v_{\mathrm{b}}$ can be assigned an effective value between 3 and 6 . Altogether, thus

$$
\frac{1}{2} \leq \frac{k_{\mathrm{b}} v_{\mathrm{b}}}{2} \leq 1
$$

B. Surface Transition Probability and Mobility. The surface process can in principle be represented by a form analogous to eq 1

$$
W_{\mathrm{s}}=\frac{\omega_{\mathrm{s}}}{2 \pi} \exp \left(-\frac{\epsilon_{\mathrm{s}}+\frac{\Delta G}{2}+\frac{E_{\mathrm{s}}}{4}}{\theta}\right)
$$

where the subscript s stands for the surface. As noted before, a driving force term $\Delta G$ has been added in the form of the free energy of an elementary endothermic PT step from the region near an $\mathrm{SO}_{3}{ }^{-}$group to an intermediate position. $\epsilon_{\mathrm{s}}$ is equivalent to $\epsilon_{\mathrm{b}}$ in eq 1 and represents the total environmental preorganization preparing the proton donor and acceptor for PT. $E_{\mathrm{s}}$ is the nuclear reorganization Gibbs free energy, equivalent to $E_{\mathrm{b}}$ in eq 1, accompanying PT from the initial prepared state to the final, similarly prepared state. While the two forms (eqs 1 and 4) are thus quite analogous, the values of the parameters are of course, in general, different. In addition, although palatable models for the surface conductivity can be constructed immediately, the specific operational models and their independent substantiation are much more elusive than models for bulk proton conductivity, for which, for example, comprehensive molecular dynamics simulation studies are available (see above).

When the distance between neighboring $\mathrm{SO}_{3}{ }^{-}$groups increases, new pathways of PT in the surface layer via an increasing number of intermediate water molecules are opened. The specific expression for the rate constant of PT between two groups then changes. These modifications lead, however, merely to an enhancement of the already predicted tendency of increasing activation energy, or decreasing rate constant, upon increasing the $\mathrm{SO}_{3}{ }^{-}$distance. This is addressed in detail in the Appendix.
The mobility can be estimated as ${ }^{28}$

$$
\mu_{\mathrm{s}}=\frac{k_{\mathrm{s}} v_{\mathrm{s}}}{2} \frac{e a_{\mathrm{s}}^{2} W_{\mathrm{s}}}{\theta}
$$

where $k_{\mathrm{s}}$ is no longer $1 / 3$. It can, generally, be expected that $k_{\mathrm{s}} v_{\mathrm{s}} / 2<k_{\mathrm{b}} v_{\mathrm{b}} / 2$ and $a_{\mathrm{s}} \sim a_{\mathrm{b}}$.

C. Mobility in the Pore. A Two-State Model. Separation of the overall proton conductivity into surface and bulk mobility mechanisms constitutes an idealization. A more comprehensive notion would be the mobility as a function of the distance from the pore walls. The mobility for a cylindrical pore model, $\mu(r)$, with $r$ being the radial coordinate, is thus

$$
\mu(r)=h(r) \frac{e a^{2}(r) W(r)}{\theta}
$$

where

$$
h(r)=\frac{k(r) v(r)}{2}
$$

where $k(r)$ and $v(r)$ are the radius-dependent geometrical factor and coordination number of PT, respectively. It is convenient and presently necessary, however, to use a two-state model. Surface mobility (index s) is assumed to prevail in boundary layers with a thickness $\delta$ of $\approx 3 \AA$, which is roughly the thickness of a water monolayer, while bulk mobility (index $b$ ) prevails in the remaining pore volume. The overall pore mobility to which experimental data could be referred is thus

$$
\begin{aligned}
\mu(r)=h_{\mathrm{s}} \frac{e a_{\mathrm{s}}^{2} W_{\mathrm{s}}}{\theta} \Theta(r-R+\delta) \Theta(R-r)+ \\
h_{\mathrm{b}} \frac{e a_{\mathrm{b}}{ }^{2} W_{\mathrm{b}}}{\theta} \Theta(R-\delta-r) \Theta(r)
\end{aligned}
$$

where $R$ is the pore radius and $\Theta$ is the step function; i.e., $\Theta(x)$ $=1$ when $x>0$ and zero otherwise.

\section{Proton "Cloud" in a Pore}

As we have discussed, a thermalized excess proton in water exists within an $\mathrm{H}^{+}-n \mathrm{H}_{2} \mathrm{O}$ cluster: $\mathrm{H}_{3} \mathrm{O}^{+}, \mathrm{H}_{5} \mathrm{O}_{2}{ }^{+}$, and $\mathrm{H}_{9} \mathrm{O}_{4}{ }^{+}$. The number $n$ fluctuates with time; i.e., the cluster dynamically changes with spontaneous breakage and recovery of hydogen bonds in the clusters and between the clusters and the remaining water. At a given time, several protons in a given volume can occupy different states with close but still different energies. These states, however, are never fixed; they fluctuate and transform one into another. Therefore, a lattice gas model for a distribution of charged particles over a limited set of sites characterized by different energies ${ }^{29}$ really may not be a better approximation than a simple Poisson-Boltzmann mean-field theory; dynamics averages out the difference between different hydrated excess proton states. There are exact solutions of this problem for a flat or cylindrical pore. We consider here specifically the cylindrical pore case, as recent neutron scattering data (V. Gordelyi, unpublished results) have provided new experimental evidence in favor of the predominance of the cylindrical pore type in swollen Nafion. Nevertheless, we are aware of the other pore geometries, lamellae and spherical micelles, that have been promoted on the basis of experimental data $^{30,31}$ as well. 
We consider a cylindrical pore of length $L_{\mathrm{p}}$ and radius $R$ with a negatively charged wall and positive countercharges distributed within it. The charge on the walls is characterized by the surface charge density $\Sigma$.

$$
\Sigma=-\frac{e}{l^{2}}=-\frac{\Lambda e}{2 \pi L_{\mathrm{p}} R}
$$

where $l$ is the "average separation" between neighboring fixed $\mathrm{SO}_{3}{ }^{-}$groups. In the second equation on the right-hand side of eq $9, \Lambda$ is the total number of $\mathrm{SO}_{3}{ }^{-}$groups (and protons) in the pore, which is assumed to be conserved. The quantity $\Lambda e / L_{\mathrm{p}}$ is, thus, an invariant of the system, giving the charge per unit length of the pore.

The pore is filled with water with a dielectric constant $\epsilon_{\mathrm{S}}$ in the surface layer and $\epsilon_{\mathrm{b}}$ in the bulk. $\epsilon_{\mathrm{b}}$ and $\epsilon_{\mathrm{s}}$ are assumed to be constant within the bulk and surface regions, respectively, but this assumption could be relaxed by using a nonlocal dielectric approach or other means. Within the surface layer, a constant, i.e., independent of the radial coordinate $r$, proton density $\rho_{\mathrm{s}}$ is, moreover, assumed

$$
\epsilon=\epsilon_{\mathrm{s}} \text { and } \rho(r)=\rho_{\mathrm{s}} \text { for } R-\delta<r<R
$$

The proton distribution $\rho(r)$ in the bulk of the pore is obtained from the Poisson-Boltzmann equation

$$
\frac{1}{\zeta} \frac{\mathrm{d}}{\mathrm{d} \zeta}\left(\xi \frac{\mathrm{d} \bar{\psi}}{\mathrm{d} \zeta}\right)=-\left(\kappa_{\mathrm{b}} R\right)^{2} \exp (-\bar{\psi})
$$

where $\zeta=r / R, \bar{\psi}=\mathrm{e} \psi / \theta$, where $\psi$ is the electrostatic potential, and $\kappa_{\mathrm{b}}=\left(e \rho_{0} / \epsilon_{0} \epsilon_{\mathrm{b}} \theta\right)^{1 / 2}=\left(4 \pi \rho_{0} \mathrm{~L}{ }_{\mathrm{B}} / e\right)^{1 / 2}$. The Bjerrum length $\mathrm{L}$ в has been introduced for later notational convenience

$$
\mathrm{L}_{\mathrm{B}}=\frac{e^{2}}{4 \pi \epsilon_{0} \epsilon_{\mathrm{b}} \theta}
$$

$L_{\text {B }}$ will be used in the following as a characteristic length scale in the system to simplify the formalism. The proton distribution for $0<r<R-\delta$ is given in analytical form by

$$
\rho(r)=\frac{\rho_{0}}{\left[1-\frac{\left(\kappa_{\mathrm{b}} r\right)^{2}}{8}\right]^{2}}
$$

where the proton concentration at the center of the pore, $\rho(r=0)$ $=\rho_{0}$, must be determined from the condition

$$
-2 \pi R \Sigma=2 \pi \int_{0}^{R} r \mathrm{~d} r \rho(r)
$$

Equation 14 states that the number of protons in a slice of the cylinder with unit thickness is equal to the number fixed of $\mathrm{SO}_{3}{ }^{-}$charges on the circumferential surface of that slice. After integration and determination of $\rho_{0}$, the following relation is obtained

$$
\rho_{0}=\frac{2 e}{\pi R^{2} L_{B}} \frac{1}{\Delta} \frac{1+\frac{\lambda}{\Delta}-\sqrt{\frac{\lambda}{\Delta}\left(2+\frac{\lambda}{\Delta}\right)-2 \lambda}}{1+2 \lambda}
$$

where the parameter $\lambda$ is

$$
\lambda=\frac{L_{\mathrm{p}}}{\Lambda \mathrm{L}_{\mathrm{B}}}
$$

The $R$-dependent variable

$$
\Delta=\left(\frac{R-\delta}{R}\right)^{2}
$$

has been introduced for further notational convenience. $\Delta$ varies between the limits 0 (only surface) and 1 (only bulk).

The proton concentration in the surface layer is determined by

$$
\rho_{\mathrm{s}}=\frac{\rho_{0}}{\left[1-\frac{\left(\kappa_{\mathrm{b}} R\right)^{2}}{8} \Delta\right]^{2}}
$$

In the limit of large $R, \rho_{0}$ is given by

$$
\rho_{0}=\frac{2}{1+2 \lambda} \frac{\Lambda e}{\pi R^{2} L_{B}}
$$

\section{Conductance of a Pore}

The conductance of a single cylindrical pore of length $L_{\mathrm{p}}$ and radius $R$ is given by

$$
G_{\mathrm{p}}=\frac{2 \pi}{L_{\mathrm{p}}} \int_{0}^{R} r \mathrm{~d} r \rho(r) \mu(r)
$$

Integration using eq 8 gives

$$
\begin{aligned}
G_{\mathrm{p}}= & G_{\mathrm{p}}^{0} \frac{\mu_{\mathrm{b}}}{\mu_{\mathrm{b}}^{0}} \frac{\Delta+\lambda\left[1-\sqrt{1+\frac{2}{\lambda} \Delta(1-\Delta)}\right]}{\left.\Delta-\sqrt{1+\frac{2}{\lambda} \Delta(1-\Delta)}\right]} \\
& \left\{1+\frac{\mu_{\mathrm{s}}}{\mu_{\mathrm{b}}}\left(1+\frac{1}{2 \lambda}\right) \frac{1-\Delta}{\Delta-\frac{1}{2}\left[1-\sqrt{1+\frac{2}{\lambda} \Delta(1-\Delta)}\right]}\right\}
\end{aligned}
$$

Here, we introduced a reference conductance

$$
G_{\mathrm{p}}^{0}=\frac{\Lambda e}{L_{\mathrm{p}}^{2}} \mu_{\mathrm{b}}^{0}
$$

representing the ideal conductance that a single cylindrical pore would obtain, if all protons in it were moving with bulk mobility $\mu_{\mathrm{b}}^{0}$ at the reference temperature $T^{0}$, determined via eqs 1 and 2 . The actual bulk mobility at temperature $T$ is determined by the ratio

$$
\frac{\mu_{\mathrm{b}}}{\mu_{\mathrm{b}}^{0}}=\frac{T}{T^{0}} \exp \left[-\frac{4 \epsilon_{\mathrm{b}}+E_{\mathrm{b}}}{4 k_{\mathrm{B}}}\left(\frac{1}{T}-\frac{1}{T^{0}}\right)\right]
$$

In the limit $\Delta \rightarrow 0$ (surface dominating), eq 21 simplifies to

$G_{\mathrm{p}} \approx G_{\mathrm{p}}^{0} \frac{\mu_{\mathrm{b}}}{\mu_{\mathrm{b}}^{0}}\left\{\Delta+\frac{\mu_{\mathrm{s}}}{\mu_{\mathrm{b}}}\left[1+\left(\frac{1}{2 \lambda}-1\right) \Delta-\frac{1}{2 \lambda}\left(1+\frac{1}{2 \lambda}\right) \Delta^{2}\right]\right\}$

and in the limit $\Delta \rightarrow 1$ (bulk dominating) to

$$
G_{\mathrm{p}} \approx G_{\mathrm{p}}^{0} \frac{\mu_{\mathrm{b}}}{\mu_{\mathrm{b}}^{0}}\left\{1-2\left(1+\frac{1}{2 \lambda}\right) \frac{\delta}{R}+2 \frac{\mu_{\mathrm{s}}}{\mu_{\mathrm{b}}}\left(1+\frac{1}{2 \lambda}\right) \frac{\delta}{R}\right\}
$$


In eq 21 , the parameters that determine the balance between surface and bulk contributions are $\mu_{\mathrm{s}}, \mu_{\mathrm{b}}$, and $\lambda$. Additional, implicit $R$ dependencies of $G_{\mathrm{p}}$ arise due to the effect of the finite pore size on $\mu_{\mathrm{s}}$ (cf. section VII) and $\epsilon_{\mathrm{b}}$. The latter may appear to be due to the spatial dielectric dispersion in confined systems or due to effects of the hydrophobic walls on the water structure. A simple parametrization of this effect is given by

$$
\epsilon_{\mathrm{b}}(R)=\epsilon_{\mathrm{b}}^{\text {free }}\left[1+(\mathrm{E}-1) \exp \left(-\frac{R}{\Lambda_{\mathrm{c}}}\right)\right]^{-1}
$$

where $E$ is the ratio of the water dielectric constant of free bulk water, $\epsilon_{\mathrm{b}}^{\text {free }}$, to the short-range dielectric constant and $\Lambda_{\mathrm{c}}(\approx 3$ $\AA$ ) is the structural correlation length in water. ${ }^{32-35}$

\section{Conductivity of a Single, Regularly Swelling Pore}

Equations 1, 4, and 8 combined with the proton carrier distribution functions in eqs 13,15 , and 16 leading to eq 21 constitute the basis for the investigation of the single-pore conductance as a function of the pore radius $R$. This kind of expression also has a direct bearing on experimental observations where such relations are available. In this section, we present model calculations for a single, ideal, cylindrical pore with a fixed number $\Lambda$ of $\mathrm{SO}_{3}{ }^{-}$groups that are regularly distributed on the pore walls. In section IX, this is converted to the overall observable conductivity of a membrane.

When $R$ is increased, the average separation $l$ between $\mathrm{SO}_{3}{ }^{-}$ groups will increase according to

$$
l=\sqrt{\frac{2 \pi L_{\mathrm{p}}}{\Lambda}} R
$$

Equation 27, together with results that we obtained in a specific study of the activation free energy in a single pore in ref 36 , gives an increase in the calculated surface activation free energy upon pore growth, i.e., increasing $l$. The reaction free energy contribution to the overall activation free energy in the surface layer increases with $l$ due to attenuated assitance of neighboring $\mathrm{SO}_{3}{ }^{-}$groups in the $\mathrm{SO}_{3} \mathrm{H}$ deprotonation process. In the following, we will denote pores in which eq 27 is valid as regular pores. It is expedient at first to discuss separately bulk and surface contributions.

The mechanism underlying the bulk mobility is expected to be weakly affected by the variation of $R$. The pore size effect on this contribution is, therefore, manifested mainly through the proton distribution, which is explicitly taken into account in eq 21 via the inclusion of eq 13 .

The mechanism underlying the surface contribution may, however, change drastically with $R$. In a dry membrane, the water structure close to the surface is rigid, with a large number of directed bonds. The dielectric constant is low due to the high concentration of $\mathrm{SO}_{3}{ }^{-}$groups and the proximity of the hydrophobic polymer walls. On one hand, this water structure produces a tendency toward a large activation free energy. On the other hand, the smaller the pores are, the smaller the average separation between neighboring $\mathrm{SO}_{3}{ }^{-}$groups is. This gives an opposite tendency toward a reduced activation free energy.

In a swollen pore, the water structure at the surface may become softer. This effect creates a tendency toward a diminished reaction free energy contribution to the surface activation free energy. However, the increasing separation $l$ between $\mathrm{SO}_{3}{ }^{-}$ groups upon swelling creates the opposite tendency toward an enhanced reaction free energy contribution. The impact of pore

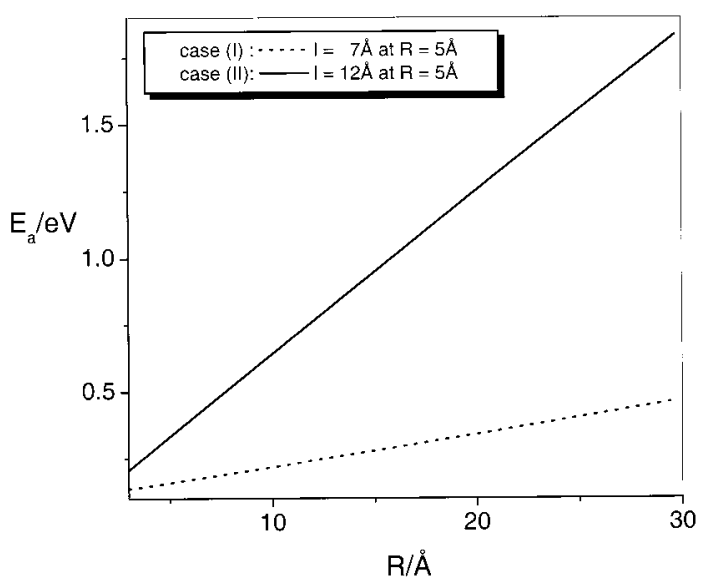

Figure 1. Activation energy of proton transfer along the pore surface, $E_{\mathrm{a}}=\epsilon_{\mathrm{s}}+\Delta G / 2+E_{\mathrm{s}} / 4$, as a function of pore radius for a regular pore, in which the separation between $\mathrm{SO}_{3}{ }^{-}$groups varies according to eq 27 with $l=7$ (case I) or $l=12 \AA$ (case II) at $R=5 \AA$.

swelling is, thus, 2-fold. Probably, the impact of $l$ will determine the overall tendency, which will then be manifested as a considerable overall increase of the surface activation free energy upon pore growth. One should however note the competing effects which inhibit a precise a priori prediction of the net tendency in the surface activation free energy. It is even harder to predict the dominating variation of the pre-exponential factor.

Instead, we have varied the parameters of the surface mobility to investigate the interplay between the different effects upon swelling. Cases where the surface contribution is insignificant are, further, compared to cases when it gives an important contribution. In practical terms, these different effects may be controlled by variation of the equivalent weight of the membrane, defined as the mass of dry polymer material per mole of ion exchange sites ( $\mathrm{SO}_{3}{ }^{-}$groups). Within the model calculations, presented here, a smaller equivalent weight, i.e., a larger concentration of $\mathrm{SO}_{3}{ }^{-}$groups, is reflected in a smaller $l$ and therefore in smaller activation free energies of surface conduction.

Electrostatic calculations on activation free energies in pores of polymer electrolyte membranes have been reported previously in ref 36. They form the basis for the subsequent analysis. The activation free energy of the bulk mobility is taken to be 0.12 $\mathrm{eV}$, determined from experimental Arrhenius plots, ${ }^{37}$ disregarding formal entropy effects. The pre-exponential factor is taken to be a fixed, adjustable number. These two quantitites are fixed in all calculations. Figure 1 shows the activation free energy of the surface mobility as a function of $R$ for distinct values of the appropriate parameters. The upper curve corresponds to separations between $\mathrm{SO}_{3}{ }^{-}$groups with a reference value $l$ of $12 \AA$ with an $R$ of $5 \AA$ and variation with $R$ according to eq 27 . The activation free energies are considerably lower and less dependent on $R$ for the lower curve when $l=7 \AA$ and $R=5 \AA$. These features reflect mutual $\mathrm{SO}_{3}{ }^{-}$assistance on the horizontal proton transfer at short $\mathrm{SO}_{3}{ }^{-}$separations. Pre-exponential factors of the surface mobility are considered to be roughly equal or slightly larger (maximum of 1 order of magnitude) than for the bulk mobility. For the explicit calculations, presented in the following, equal pre-exponential factors will be used for both mechanisms.

All subsequently presented conductance data will be scaled relative to the parameter $G_{\mathrm{p}}^{0}$, defined in eq 22 . The corresponding reference mobility $\mu_{\mathrm{b}}^{0}$ is specified at temperature $T^{0}$. 
Using the definitions in eqs $1,2,9$, and $22, G_{\mathrm{p}}^{0}$ is given by

$$
G_{\mathrm{p}}^{0}=\frac{k_{\mathrm{b}} v_{\mathrm{b}} \omega_{\mathrm{b}} e^{2}}{2 k_{\mathrm{B}}} \frac{R}{L_{\mathrm{p}}} \frac{a_{\mathrm{b}}^{2}}{l^{2}} \frac{1}{T^{0}} \exp \left(-\frac{\epsilon_{\mathrm{b}}+\frac{E_{\mathrm{b}}}{4}}{k_{\mathrm{B}} T^{0}}\right)
$$

revealing important dependencies on pore geometry ( $R$ and $L_{\mathrm{p}}$ ), microscopic distances $\left(a_{\mathrm{b}}\right.$ and $\left.l\right)$, and $T^{0}$. We fix the reference temperature $T^{0}$ at $373 \mathrm{~K}$ and use, for an order-of-magnitude estimate, the following parameter values: $k_{\mathrm{b}} v_{\mathrm{b}} / 2=1$ (cf. eq 3), $\omega_{\mathrm{b}}=5 \times 10^{14} \mathrm{~s}^{-1}$ (cf. refs 28 and 38), $a_{\mathrm{b}}=3 \AA, L_{\mathrm{p}}=50$ $\AA, \epsilon_{\mathrm{b}}+E_{\mathrm{b}} / 4=0.12 e \mathrm{eV}$. Due to the assumption of charge conservation, i.e., conservation of $\Lambda$ (cf. eq 9), within a single pore upon swelling, the ratio $R / l^{2}$ is independent of $R$. Two cases with different surface densities of fixed charges will be considered: (I) $l=7 \AA$ at $R=5 \AA$ (Figures 2 and 4) and (II) $l=12 \AA$ at $R=5 \AA$ (Figure 3 ). With these values, we obtain the estimates

$$
G_{\mathrm{p}}^{0} \approx 5.5 \times 10^{-7} \mathrm{~S}(\mathrm{I}) \text { and } 1.8 \times 10^{-7} \mathrm{~S}(\mathrm{II})
$$

Single-pore Arrhenius plots $\left[\log _{10}\left(G_{\mathrm{p}} T / G_{\mathrm{p}}^{0} T^{0}\right)\right.$ vs $\left.1000 / T\right]$ and the $R$ dependence of $G_{\mathrm{p}}$ (normalized to $G_{\mathrm{p}}^{0}$ ) corresponding to different parametrizations of the surface mobility are shown in Figures 2-4. Figure 2, obtained with the lower curve of Figure 1 (case I), represents a large surface mobility with a small pore size. However, the surface mobility decreases rapidly upon pore growth due to the increasing activation free energy. The total conductance plotted as a function of $R$ at different temperatures in panels $\mathrm{b}(273 \mathrm{~K})$ and $\mathrm{c}(373 \mathrm{~K})$ has, interestingly, a pronounced minimum at $R \approx 8 \AA$. When the activation free energy of the surface mobility exceeds $\approx 0.24 \mathrm{eV}$, corresponding to radii of $>10 \AA$, the surface mobility becomes insignificant.

Figure 3, obtained with the upper curve of Figure 1 (case II), represents the case of a small surface contribution due to large $\mathrm{SO}_{3}{ }^{-}$separation. The surface contributes insignificantly to the overall conductance (cf. panels b and $c$ of Figure 3). The conductance increases monotonically with pore size in this case.

In contrast to the correlations shown in Figures 2 and 3, which were calculated with the $R$-dependent surface activation energies of Figure 1, the correlations depicted in Figure 4 have been calculated with the activation energy of the surface contribution fixed. In this case, the conductance increases monotonically with increasing $R$, since the dependence of the surface mobility on $R$ is much weaker than in the previous cases.

In the various plots of $G_{\mathrm{p}}(R)$ at $373 \mathrm{~K}$, the conductance at the largest depicted radii amounts to about $75 \%$ of the ideal conductance $G_{\mathrm{p}}^{0}$.

\section{Discussion of the Single-Pore Results}

Our calculations show that there are definitely two contributions to the proton conductance of a single pore: one contribution dominates in the middle of the pore and proceeds in a manner similar to that of bulk water, and the other contribution dominates near the pore surface along the array of $\mathrm{SO}_{3}{ }^{-}$groups.

The surface mobility is usually considerably smaller and has a higher activation free energy than bulk mobility. This may, however, be partially compensated by the accumulation of protons in the surface region. Squeezing the pore to smaller dimensions will diminish the size of the bulklike portion of water, and practically all the proton transport will then be surface-like in sufficiently narrow pores.
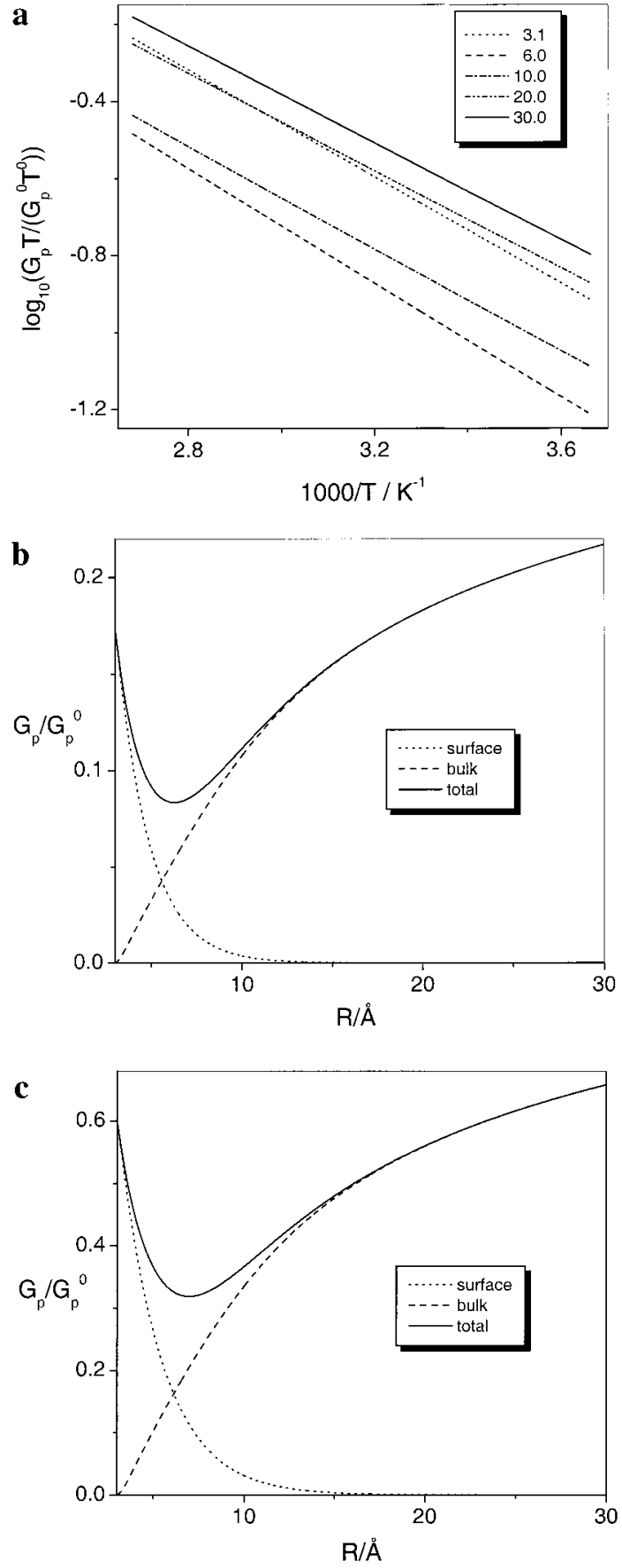

Figure 2. High surface mobility in small pores. Arrhenius plots $\left\{\log _{10}\left[G_{\mathrm{p}} T /\left(G_{\mathrm{p}}^{0} T^{0}\right)\right]\right.$ vs $\left.1000 / T\right\}$ of single-pore conductivity at various radii (a) and dependence of pore conductivity (normalized to $G_{\mathrm{p}}^{0}$ ) on radius at 273 (b) and $373 \mathrm{~K}$ (c), obtained from eq 21 . The activation energy of the bulk mobility was fixed as $0.12 \mathrm{eV}$. For the surface activation energy, the $R$-dependent parametrization with $l=7 \AA$ at $R$ $=5 \AA$ (cf. Figure 1) was used (case I, for which the numerical estimate gives $G_{\mathrm{p}}^{0} \approx 5.5 \times 10^{-7} \mathrm{~S}$ ). The pre-exponential factor was assumed to be the same as for both mobility mechanisms. The conductivity is high at small $R$ due to the excellent surface mobility. With increasing $R$ values, the surface mobility decreases rapidly (see panels b and c), whereas the bulk contribution increases due to the increasing fraction of bulk protons.

The families of Arrhenius relations at various $R$ values in Figures 2-4 look rather different and depend on whether the surface contribution is significant. In a regularly swelling pore, in which $l$ increases with $R$ according to eq 27, the potential wells in which the surface protons are trapped become deeper 

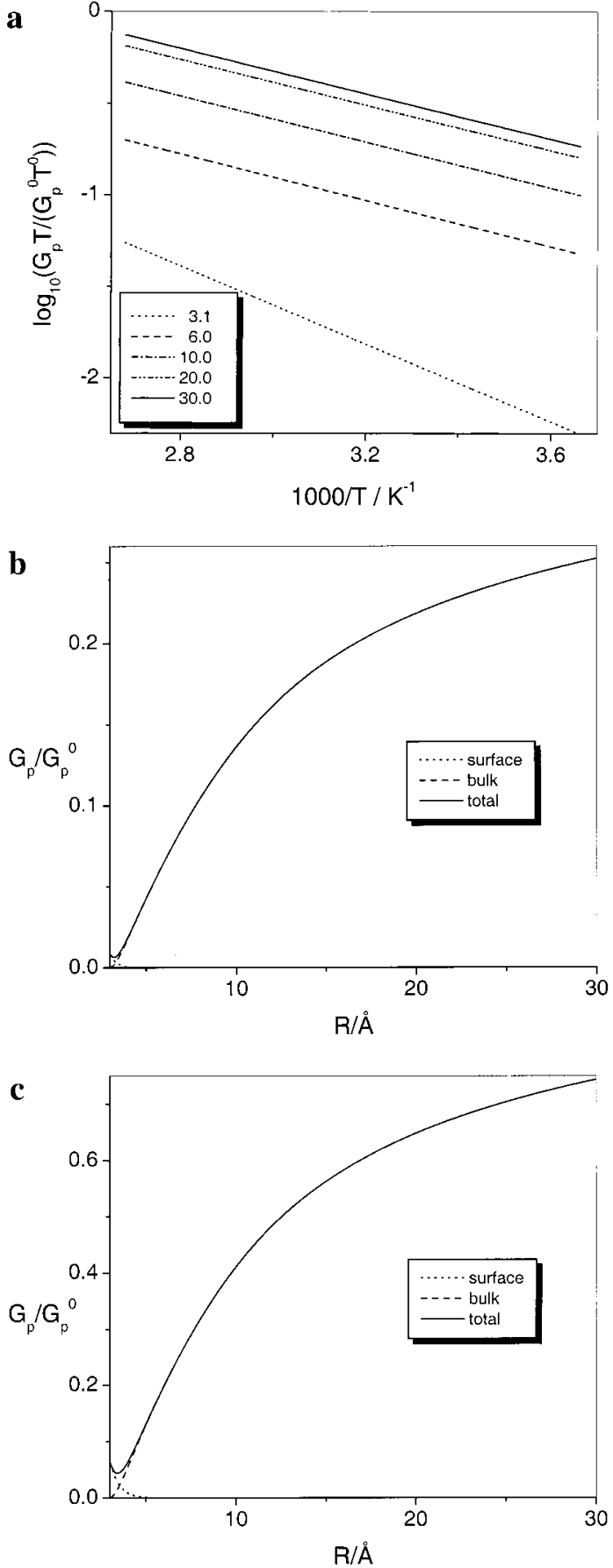

Figure 3. Low surface mobility in small pores. The same set of plots as in Figure 2. For the surface activation energy, the $R$-dependent parametrization with $l=12 \AA$ at $R=5 \AA$ (cf. Figure 1) was used (case II, $G_{\mathrm{p}}^{0} \approx 1.8 \times 10^{-7} \mathrm{~S}$ ). Overall, due to the high activation energy of the surface mobility, the conductivity is mainly determined by the bulk transport. The surface mobility contributes considerably only in the range of very small $R$.

with increasing $R \cdot{ }^{36}$ Surface protons therefore become immobilized at the $\mathrm{SO}_{3}{ }^{-}$groups upon swelling, whereas the fraction of highly mobile bulk protons increases continuously.

Generally, a pore with a high surface density of $\mathrm{SO}_{3}{ }^{-}$groups and, thus, a higher proton concentration performs better. Due to a considerable surface contribution, the conductance can be excellent even at small pore sizes. Since, however, the surface mobility rapidly decreases with increasing $R$, whereas the bulk conductance increases due to an increasing fraction of bulk
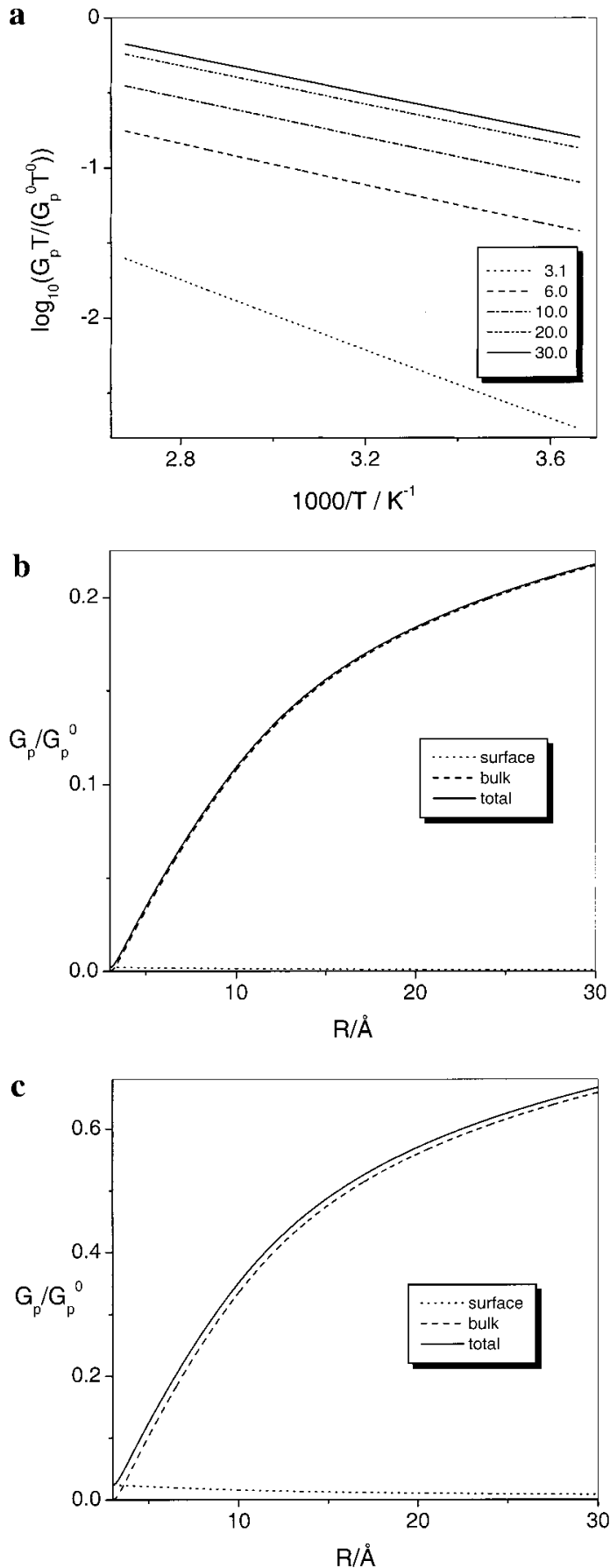

Figure 4. $R$-independent surface mobility. The same set of plots as in Figure 2 with density of $\mathrm{SO}_{3}{ }^{-}$groups corresponding to $l=7 \AA$ at $R=$ $5 \AA$. For the surface activation energy, a fixed value $0.24 \mathrm{eV}$ was used.

protons, a pronounced minimum in conductance at intermediate pore sizes appears. Overall, the conductance is higher due to a higher proton concentration, as indicated by the corresponding value of $G_{\mathrm{p}}^{0}$.

A regular pore with a high surface density of $\mathrm{SO}_{3}{ }^{-}$groups could perform equally well at low and high water contents (cf. Figure 2). Due to the larger value of $G_{\mathrm{p}}^{0}$, this kind of pore will be superior in conductance compared to the kind of pore used to generate Figure 3. A low degree of swelling could then be sufficient for good overall performance, characterized by a good conductance. Moreover, due to the prevalence of the surface mobility, permeation of methanol and water would probably be suppressed under these conditions. These properties of the single pore would be directly related to characteristics of the 
whole membrane, if the appropriate type of pores would dominate in the percolating network of membrane pores.

The presence of a considerable fraction of irregular pores with large $\mathrm{SO}_{3}{ }^{-}$group separations could, however, hamper the overall performance of the membrane. In this case, a bundle of parallel, equally performing regular pores is not a good model of the membrane. In particular, in the dry membrane, there may be a significant fraction of poorly conducting irregular pores, the heterogeneities of which would require separate attention.

As demonstrated above, the EW and the density of $\mathrm{SO}_{3}{ }^{-}$ groups per unit pore length may affect not only the proton concentration but also the competition between the surface and bulk contributions, with a considerable effect on the membrane performance. A lower EW may, thus, give a higher surface density of $\mathrm{SO}_{3}{ }^{-}$groups at the pore surfaces, resulting in an enhanced surface contribution.

\section{Basic Equations for Conductance of a Membrane}

The overall membrane conductance is determined by the interplay between the charge distribution inside pores, the mobility mechanisms, and the membrane porous structure. The effect of pore structure will be discussed in this section. Two major impacts of the pore structure variations on electrical conductance can be distinguished: the variation of the singlepore cross-sectional area, available for proton conduction; and the orientation and topology of conducting pores in the network. Protons are released to the enclosed bulk from the interior pore surface. Therefore, the fixed surface density of $\mathrm{SO}_{3}{ }^{-}$groups directly influences the concentration of protons in the bulk. There is, thus, no intrinsic, isotropic bulk conductivity of the pore-filling medium (water). The latter would be observed if pores were filled with a bulk electrolyte solution at a fixed concentration. This principal difference hampers the straightforward transfer of results of well-known studies on conductivity in porous media ${ }^{13,39,40}$ to polymer electrolyte membranes. As we will see below, even simple models lead to relations between conductivity and porosity more complex than Archie's law. ${ }^{41}$

A polymer membrane differs from a rigid porous body, such as a sandstone; there is no fixed, pre-existing porosity in it. In the membrane, the porous structure is formed upon the uptake of water. Water is the pore former, not only a pore filler. The pore network can, thus, be envisaged as an "evolving organism" that reorganizes continuously during swelling.

In the past, there have been extensive studies about the evolution of pore geometry in this network. ${ }^{11,27,30,42,43}$ However, no single accepted view of membrane structure has emerged. Probably, no simple geometry will give an appropriate description. Instead, it is most likely that the water fractions can be considered to form tortuous pathways with ramified boundaries. Nonetheless, this highly interconnected porous structure may be subdivided, at least for model purposes, into elementary segments resembling lamellae, cylinders, or spheres. According to recently obtained experimental data, ${ }^{42}$ cylindrical pores prevail in the swollen membrane. Upon dehydration, the number of pore constrictions increases, possibly leaving structures that look like networks of spherical pores connected by narrow cylindrical channels.

The simplest possible calculation of the sample conductance as a function of water content would rest on a model of parallel identical straight tubes of length $L_{\mathrm{p}}$ with a uniform cylindrical cross section. ${ }^{39}$ Within such an approach, the single pore properties completely carry over to the membrane (simplistic model). As a next step toward a more sophisticated description, a distribution of diameters of parallel straight tubes can be considered (parallel model). Another step of complication is to allow nonuniform tube cross sections, i.e., to virtually cut the tube into $n$ cylindrical segments of equal lengths $l_{\mathrm{p}}\left(=L_{\mathrm{p}} / n\right)$ with a distribution in segment diameters (serial model). ${ }^{44}$ In calculations of transport properties (conductivity and permeability) across the membrane, the fact that on average only one-third of all the tubes should be pointing in each of the three spatial directions must be taken into account ${ }^{39}$ (provided that the membrane is isotropic).

Finally, the pore space model, which should provide the closest approach to reality, consists of a three-dimensional system of interconnected tubular segments of various lengths and diameters (network model). Effective medium theory (the abbreviation EMT will be used hereafter) could be used as a good approximation for a calculation of the conductivity of such an interconnected network. ${ }^{11,45,46}$

Parallel and serial type models require the incorporation of a tortuousity factor $\tau^{2}$ the tortuousity factor $\tau$, as defined by von Karman in 1937, is the square of the ratio of the hypothetical tortuous capillary length, $L_{\mathrm{p}}$, to the thickness of the porous membrane in the direction of macroscopic flow, $L\left[\tau=\left(L_{\mathrm{p}}\right)\right.$ $\left.\left.L)^{2}\right]^{39}\right\}$ to account for a possibly curved form of the channels. The non-interconnected, one-dimensional model variants (serial type models) tend to underestimate the transport properties. ${ }^{39}$ Furthermore, they are very sensitive to details of the pore size distribution for the smallest pore sizes due to the "bottleneck effect": a single poorly conducting segment can limit the overall transport, since in one dimension it cannot be bypassed.

Our calculations of the membrane conductance will be based on serial and network type models. In the serial model, ${ }^{44}$ the pore space is formed by parallel tortuous channels, each of which is assumed to consist of elementary cylindrical segments with distributed radii according to a given pore size distribution. This pore size distribution evolves with the water content. An extension to a three-dimensional network with interconnected elementary segments will be discussed in terms of the effective medium theory. ${ }^{46}$

A. Integral Relations. We begin with an outline of how the pore size distribution and its evolution are related to the water content and to the membrane conductance in the serial and network type models.

The mass of dry membrane material and the total number of parallel tubes $N$ are assumed to be constant upon swelling. Furthermore, the effective tube length $L_{\mathrm{p}}$ along the thickness of the membrane is assumed to be fixed. The water content can then be expressed in terms of the volume or weight fraction

$$
w_{\mathrm{V}}=\frac{V_{\mathrm{w}}}{V_{\mathrm{p}}+V_{\mathrm{w}}} \text { or } w_{\mathrm{M}}=\frac{M_{\mathrm{w}}}{M_{\mathrm{p}}+M_{\mathrm{w}}}
$$

where $M_{\mathrm{w}}$ and $V_{\mathrm{w}}$ are the mass and the volume of the water fraction in the membrane, respectively, and $M_{\mathrm{p}}$ and $V_{\mathrm{p}}$ are the mass and the volume of the dry polymer material, respectively. Due to the fixed tube length $L_{\mathrm{p}}$, the total water volume is given by

$$
V_{\mathrm{w}}=N \pi\left\langle R^{2}\right\rangle L_{\mathrm{p}}, M_{\mathrm{w}}=V_{\mathrm{w}} \rho_{\mathrm{w}}
$$

where $\rho_{\mathrm{w}}$ is the density of water. The mean square radius of pores (cylindrical segments) is given by

$$
\left\langle R^{2}\right\rangle=\int_{0}^{\infty} \mathrm{d} R R^{2} g(R)
$$

where $g(R)$ is the "pore size distribution" function, abbreviated 
as psd hereafter. Each element of the linear chain has a probability density $g(R)$ to have the radius $R$. The shape of the psd and its evolution upon water uptake determine the water volume fraction. The general expression of the water content is, thus, given by

$$
w=\frac{\pi\left\langle R^{2}\right\rangle}{A+\pi\left\langle R^{2}\right\rangle}
$$

Here, the effective "area" $A$ is a parameter of the dry membrane, a normalized cross section of the polymer part. It is defined differently, subject to whether the water content is given in terms of the volume fraction $\left[A_{\mathrm{V}}=V_{\mathrm{p}} /\left(N L_{\mathrm{p}}\right)\right]$ or the mass fraction $\left[A_{\mathrm{M}}=M_{\mathrm{p}} /\left(N L_{\mathrm{p}} \rho_{\mathrm{w}}\right)=A_{\mathrm{v}} \rho_{\mathrm{p}} / \rho_{\mathrm{w}}\right]$.

In the serial model of cylindrical segments, identical in length, the membrane conductance is given by ${ }^{39}$

$$
G_{\mathrm{M}}=\frac{N}{3 n} G_{\mathrm{p}}^{\mathrm{e}}
$$

where $G_{\mathrm{p}}^{\mathrm{e}}$, an effective conductance of a segment, is defined by

$$
G_{\mathrm{p}}^{\mathrm{e}}=\left\{\int_{0}^{\infty} \mathrm{d} R\left[G_{\mathrm{p}}(R)\right]^{-1} g(R)\right\}^{-1}
$$

The factor 3 in the denominator is due to the fact that only one-third of all pores point in the spatial direction of macroscopic flow.

In the three-dimensional, multiply interconnected random resistance network, the system of Kirchhoff equations for current densities through all nodes and for potential drops between the nodes must be solved. This complex task can be handled exactly only by means of numerical simulations. An approach toward this problem, developed by Kirkpatrick, ${ }^{45}$ is based on EMT. Here, the random resistance network is replaced with an effective network in which the elementary conductances $G_{\mathrm{p}}(R)$, distributed according to the psd $g(R)$, are replaced with identical effective conductances $G_{\mathrm{p}}^{\mathrm{e}}$. The value of the latter is determined self-consistenly from the integral equation ${ }^{46,47}$

$$
\int_{0}^{\infty} \frac{\left[G_{\mathrm{p}}^{\mathrm{e}}-G_{\mathrm{p}}(R)\right] g(R) \mathrm{d} R}{G_{\mathrm{p}}(R)+(z / 2-1) G_{\mathrm{p}}^{\mathrm{e}}}=0
$$

where $z$ is the network coordination number (the number of bonds meeting at a node), and $z=6$ for a cubic lattice [a refined approximation suggested by Bernasconi and Wiesmann ${ }^{48}$ incorporates correlations among water-filled bonds meeting at a node; however, it leads to an equation equivalent to eq 34 if one replaces $z / 2-1$ with a dimension-dependent parameter, and in complex disordered media with ill-defined coordination numbers, this parameter can be treated as a fitting parameter which reproduces the correct percolation behavior ${ }^{11}$ ]. Equation 34 establishes the link between the effective conductance $G_{\mathrm{p}}^{\mathrm{e}}$ of an individual cylindrical segment and the structural characteristics of the pore network, i.e., the psd and $z$. The overall effective network conductance $G_{\mathrm{M}}$ is proportional to $G_{\mathrm{p}}^{\mathrm{e}}$. For the serial model, corresponding to $z=2$, this proportionality factor is given by $N / 3 n$ (cf. eq 32), where the term in the denominator accounts for the tortuousity of the tubes, since $n$ stands for the ratio of actual tube length $L_{\mathrm{p}}$ to the membrane thickness. For the network model, the proportionality factor depends on the interconnetivity and tortuousity of the pore space in a somewhat more complex way.

Equations 30-34 form the general basis on which the calculation of relations between $G_{\mathrm{M}}$ and $w$ is performed.
Equations 30 and 31 establish the relation between the psd and $w$. The psd acquires new shapes with the variation of $w$. Note that the definitions used within the calculation of $w$ are independent of the system dimensionality and the specific model variant. By means of eqs $32-34, G_{\mathrm{M}}$ is related to the psd.

To calculate $G_{\mathrm{M}}(w)$, we need to specify $G_{\mathrm{p}}(R)$ and $g(R) . G_{\mathrm{p}}(R)$ was calculated in section VI. The procedure of the generation of a suitable psd which continuously evolves with $w$ is described in the next subsection.

B. Model of Pore Space Formation during Swelling and Deswelling. The requirements for the model of pore space formation that we will introduce here are as follows. The psd should closely resemble experimentally observed psds of polymer electrolyte membranes such as Nafion and its relatives. The data in ref 49 are well-approximated by a log-normal psd, ${ }^{50}$ a typical psd of ultrafiltration membranes. ${ }^{51}$ Moreover, the model should allow the straightforward incorporation of single pore information, i.e., $G_{\mathrm{p}}(R)$.

Particularly convenient in this respect is the so-called bond shrinkage/expansion model, ${ }^{14}$ constructed subject to the following algorithm. Consider a single straight cylindrical tube with a uniform cross section. This single tube of length $L_{\mathrm{p}}$ is now subdivided into $n$ cylindrical segments of length $l_{\mathrm{p}}$ $\left(=L_{\mathrm{p}} / n\right)$. At each step of the iterative procedure, choose randomly a single segment $i$ and multiply its radius by a fixed factor $t$,

$$
R_{i} \rightarrow t R_{i}
$$

Depending on $t$, the water fraction will shrink $(t<1)$ or expand $(t>1)$. This model resembles a regular, continuous (de-)swelling process. It is straightforward and simple, and at any stage of the swelling procedure, it allows us to relate $w$, $g(R)$, and $G_{\mathrm{M}}$, using $G_{\mathrm{p}}(R)$ as an input. For the serial pore model, analytical expressions (or at least integral relationships) for $w$ and $G_{\mathrm{M}}$ can be obtained. Moreover, the procedure can be extended to three-dimensional interconnected networks.

It will be shown in section IX.E that the psd evolving out of the initial configuration after $M$ elementary procedures is a lognormal distribution. The parameters which control its shape are the shrinkage/expansion parameter $t$ and the number $M$. The closer $t$ is to 1 , the narrower the psd is. In the limit $t \rightarrow 1$, the psd will approach a $\delta$ function-like distribution, equivalent to the simplistic pore space model.

The model provides various options. If, for example, $t=0$, segments completely vanish at a single step, the usual bondpercolation problem with finite conductance threshold at $w>$ 0 will be recovered. ${ }^{53}$ Otherwise, at $t>0$, the system will have the conduction threshold at $w=0$. Further options can be introduced by additional constraints. For instance, one may fix an initial monodisperse psd for the dry system and then allow each segment to swell exactly one time by an equal amount. This resembles the two-state model, where pores are either dry with poor residual conductivity or swollen to the equilibrium radius in the water-filled state with good conductivity. The latter problem was studied in detail in ref 11 .

C. Analytical Expressions. The initial distribution of the cross sections of the cylindrical segments of length $l_{\mathrm{p}}$ is given by

$$
A_{i, 0}=\pi R_{i, 0}^{2}, i=1, \ldots, n
$$

where the first index $i$ denotes the number of the element in the ensemble and the second index denotes the number $M$ of iterations performed on this particular segment. The initial distribution corresponds to $M=0$. Since $l_{\mathrm{p}}$ is fixed, the ensemble 
average of $A_{i, M}$ will determine the mean water volume in a tube

$$
v_{\mathrm{w}}=L_{\mathrm{p}}\left\langle A_{i, M}\right\rangle_{n}=L_{\mathrm{p}} \pi\left\langle R_{i, M}{ }^{2}\right\rangle_{\mathrm{p}}
$$

where the terms in broken brackets denote an ensemble average over all segments.

After $M$ statistically independent elementary shrinking or expanding processes have been performed, a new distribution of average cross sections, $\bar{A}_{i, M}$, will emerge. The probability for any particular segment $i$ to shrink or expand exactly $m$ times $(0 \leq m \leq M)$ is given by the binomial distribution

$$
P(m)=\frac{M !}{(M-m) ! m !}\left(\frac{1}{n}\right)^{m}\left(\frac{n-1}{n}\right)^{M-m}
$$

The average cross section of that segment is then given by

$$
\bar{A}_{i, M}=A_{i, 0} \sum_{m=0}^{M} t^{2 m} P(m)=A_{i, 0}\left(1+\frac{t^{2}-1}{n}\right)^{M}
$$

and the new average cross section of the one-dimensional network is

$$
\pi\left\langle R^{2}\right\rangle_{n}=\left\langle\bar{A}_{i, M}\right\rangle_{n}=\left\langle A_{i, 0}\right\rangle_{n}\left(1+\frac{t^{2}-1}{n}\right)^{M}
$$

This expression will also be valid in three dimensions.

The resistance of the serial array of conductances in one tube of length $L_{\mathrm{p}}$ is obtained from

$$
\mathrm{R} \quad \text { tube }=n\left\langle\sum_{m=0}^{M}\left[G_{\mathrm{p}}\left(t^{m} R_{i}\right)\right]^{-1} P(m)\right\rangle_{n}
$$

Equation 38, inserted into eq 31, and eq 39 establish the relation between $w$ and $G_{\mathrm{s}}[=N /(3 \mathrm{R}$ tube $)]$. This relation is determined by the initial psd, i.e., by the set $R_{i, 0}$, by $G_{\mathrm{p}}(R)$, by the parameter $t$, and by the number of processes $M$.

D. Analytical Solutions. Power Law Dependence. There are analytical expressions for $G_{\mathrm{s}}(w)$, if $G_{\mathrm{p}}(R)$ follows a power law

$$
G_{\mathrm{p}}(R)=k_{1} R^{\mu}
$$

with a constant $k_{1}$. The summation in eq 39 then gives for the single tube resistance

$$
\mathrm{R} \quad \text { tube }=\frac{n}{k_{1}}\left\langle R_{i, 0}{ }^{-\mu}\right\rangle_{n}\left(1+\frac{t^{-\mu}+1}{n}\right)^{M}
$$

For $t<1$, in the thermodynamic limit $(n \rightarrow \infty)$ and at small $w$, one finds after elementary algebra a power law

$$
G_{\mathrm{M}}(w) \propto g w^{\xi}
$$

with the exponent

$$
\xi=\frac{1}{t^{2}} \frac{t^{2}-t^{2-\mu}}{t^{2}-1}
$$

and the proportionality constant

$$
g=\frac{N}{3 n} k_{1}\left(\frac{A}{\pi R_{i, 0}^{2}}\right)^{\xi}
$$

Since the exponent is determined by the parameters $t$ and $\mu$, it is, thereby, affected by the shape of the psd. If, in particular, the pores were filled with a homogeneous bulk electrolyte of fixed, $R$-independent conductivity, then $\mu=2$ and $G_{\mathrm{M}}(w) \propto$ $w^{\left(1 / t^{2}\right)}$. These proportionality relations indicate that one should expect to find an effect of hysteresis between swelling $(t>1)$ and drying $(t<1)$ processes in $G_{\mathrm{M}}(w) . t>1$ produces a tendency toward convex relations $G_{\mathrm{M}}(w)$ (with an exponent $\xi$ $<1$ ), whereas $t<1$ gives concave forms $(\xi>1)$. For $t \approx 1$, corresponding to a very narrow, $\delta$ function-like psd, one would expect a simple proportionality $G_{\mathrm{M}} \propto w$ and a small hysteresis.

Membrane with Residual Surface Conductivity. Suppose now an initial distribution

$$
R_{i, 0}=\delta
$$

corresponding to a residual water content

$$
w^{\mathrm{res}}=\frac{\pi \delta^{2}}{A+\pi \delta^{2}}
$$

and consider the membrane swelling process $t>1$. In the limit of small extra water contents, i.e., $w$ close to $w^{\text {res }}$ and $R_{i, \mathrm{M}}$ close to $\delta$ for all segments, $G_{\mathrm{p}}(R)$ is given by eq 24 which can be written in the form

$G_{\mathrm{p}}(R)=G_{\mathrm{p}}^{\mathrm{res}}\left[1+k_{2}\left(1-\frac{2}{t^{m}}+\frac{1}{t^{2 m}}\right)\right], k_{2}=\frac{\mu_{\mathrm{b}}}{\mu_{\mathrm{s}}}+\frac{1}{2 \lambda}-1$

Here, $G_{\mathrm{p}}^{\mathrm{res}}\left(=\left(\Lambda e / l_{\mathrm{p}}^{2}\right) \mu_{\mathrm{s}}\right)$ is the residual conductance due to the surface contribution. After insertion of $G_{\mathrm{p}}(R)$ into eq 39, straightforward algebraic analysis gives the following approximation

$$
\begin{array}{r}
G_{\mathrm{M}}(w) \approx \frac{N}{3 n} G_{\mathrm{p}}^{\mathrm{res}}\left\{1+k_{2}\left[\frac{t-1}{t^{2}(t+1)}\left(\frac{w}{w^{\text {res }}}-1\right)-\right.\right. \\
\left.\left.\frac{1}{2} \frac{t^{4}-2 t-1}{t^{4}(t+1)^{2}}(w-1)^{2}\right]\right\}
\end{array}
$$

which is the law expected at small $w \rightarrow w^{\text {res }}$. This describes a linear dependence $G_{\mathrm{M}}(w)$ for $w \rightarrow w^{\text {res }}$. The maximum slope of the linear term is obtained for $t=1.62$. For $t$ close to 1 , the quadratic term will become dominating. The behavior of $G_{\mathrm{M}}(w)$ in the limit of small water contents can, thus, provide information about the pore space evolution and about the character of the psd. Figure 5 depicts, how the parameter $t$ of pore space evolution affects $G_{\mathrm{M}}(w)$ in the limit $w \rightarrow w^{\text {res }}$. The closer $t$ is to 1 , the more concave is $G_{\mathrm{M}}(w) . t \approx 1.5$ gives a more or less linear relation over the plotted range.

E. PSD Continuum Limit. Here, we present expressions for the psd in the continuum limit. We consider a monodisperse initial configuration with $R_{i, 0}=R_{0}$ for all pores. In the limit $n$ and $M \rightarrow \infty$, the binomial probability distribution $P(m)$, eq 36 , approaches a Gaussian distribution for $M / n \gg 1$

$$
P(m)=\frac{1}{\sqrt{2 \pi \bar{m}}} \exp \left[-\frac{(m-\bar{m})^{2}}{2 \bar{m}}\right], \bar{m}=\frac{M}{n}
$$

Note that, in the limit of small $w$, i.e., for $M / n=0$ (1), the continuuum limit corresponds to a Poisson distribution

$$
P(m)=\frac{\bar{m}^{m}}{m !} \exp (-\bar{m})
$$

This limit, however, was already studied by means of the analytical expressions in the previous subsection. In the further 


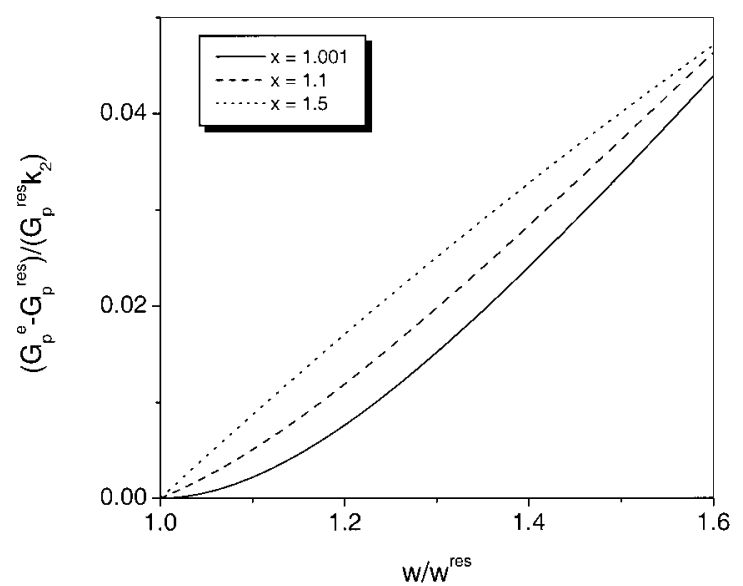

Figure 5. Effect of $t$, the parameter characterizing the skewness of the psd, on the membrane conductivity $G_{\mathrm{s}}(w)$ in the limit of small water contents $w$ approaching the residual value $w^{\text {res }}$. On the abscissa, the difference between the effective $G_{\mathrm{p}}^{\mathrm{e}}(w)$ and residual pore conductance $G_{\mathrm{p}}^{\text {res }}$ is plotted, normalized to $G_{\mathrm{p}}^{\text {res }} k_{2}$. For $t \approx 1$, i.e., for a narrow psd, the linear term becomes small. $t \approx 1.5$ gives a more or less linear relation over the plotted range.

analysis of this subsection, we will, therefore, concentrate on the Gaussian distribution.

The radius of a pore is given by $R=R_{0} t^{m}$, and the radius probability distribution is thus given by

$$
\begin{aligned}
g(R) & = \pm\left.\frac{\mathrm{d} m}{\mathrm{~d} R} P(m)\right|_{m \rightarrow R} \\
& = \pm \frac{1}{\sqrt{2 \pi \bar{m}}} \frac{1}{\ln (t)} \frac{1}{R} \exp \left\{-\frac{\left[\frac{\ln \left(R / R_{0}\right)}{\ln (t)}-\bar{m}\right]^{2}}{2 \bar{m}}\right\}
\end{aligned}
$$

(with a $+\operatorname{sign}$ for $t>1$ and - otherwise) and the differential water volume distribution is given by

$$
\begin{gathered}
v_{\mathrm{w}}(R)=N L_{\mathrm{p}} \pi R^{2} g(R) \\
= \pm N L_{\mathrm{p}} R_{0} \sqrt{\frac{\pi}{2 \bar{m}} \exp \left\{\frac{\bar{m}}{2} \ln (t)[2+\ln (t)]\right\}} \exp \left[-\frac{\left(\ln \frac{R}{\alpha}\right)^{2}}{s}\right]
\end{gathered}
$$

where $\alpha=R_{0} \exp \{\bar{m} \ln (t)[1+\ln (t)]\}$ and $s=2 \bar{m}[\ln (t)]^{2} . t$ and $\bar{m}$ determine the width, the location of the maximum, and the norm (i.e., the water volume). The total water volume is given by

$$
\begin{aligned}
V_{\mathrm{w}}=N L_{\mathrm{p}} \pi R_{0}^{2} \exp \{\bar{m} \ln (t)[1+ \\
\ln (t)]\} \frac{1}{2}\left(\operatorname{erf}\left\{\sqrt{\frac{\bar{m}}{2}}[1+2 \ln (t)]\right\}+1\right)
\end{aligned}
$$

where the integration over $R$ was taken in the interval from $R_{0}$ to $\infty$ for $t>1$ (swelling) or from 0 to $R_{0}$ for $t<1$ (drying).

The distribution given by eq 51 is a log-normal distribution. In refs 49 an 50, it was found that this kind of psd closely resembles experimental porosity data for Nafion and similar membranes.

\section{Results on Membrane Conductance}

In this section, the effect of the psd on $G_{\mathrm{M}}(w)$ will be studied. We fix the initial configuration for the dry membrane as a monodisperse psd with pore radius $R_{0}=\delta=3 \AA$ and consider the two opposing cases of a very narrow $(t=1.002)$ and a very broad $(t=1.1)$ psd. We will assume that saturation will be reached at a water volume fraction $w=0.4 .{ }^{49}$ On the basis of experimental data from neutron scattering ${ }^{31,42}$ and permeability ${ }^{52}$ and porosity measurements, ${ }^{49}$ we assume that the mean radius corresponding to saturation $\sqrt{\left\langle R^{2}\right\rangle}=30 \AA$. The phenomenological parameter $A$ in eq $31=\pi \times 1350 \AA^{2}$.

A. Limiting Cases. Before we present numerical data for the pore evolution model, we will discuss two limiting cases: (1) a homogeneous membrane with a monodisperse ( $\delta$ functionlike) psd, corresponding to the simplistic model, and (2) a heterogeneous membrane in which each cylindrical segment is allowed to swell exactly one time from its residual radius $R_{0}$ of $3 \AA$, having a residual conductance $G_{\mathrm{p}}^{\text {res }}$, to the water-swollen radius $R_{\mathrm{w}}$ of $30 \AA$ with a saturation conductance $G_{\mathrm{p}}^{\mathrm{s}}$. Case 2 corresponds to the so-called two-state model (bidisperse), studied previously in detail in ref 11 .

For case $1, G_{\mathrm{M}}(w)$ is given by

$$
G_{\mathrm{M}}(w)=\left.\frac{N}{3 n} G_{\mathrm{p}}(R)\right|_{R \rightarrow w}
$$

with $G_{\mathrm{p}}(R)$ taken from eq 21 . Here, $w$ is expressed through $R$ according to

$$
w=\frac{\pi R^{2}}{A+\pi R^{2}}
$$

For case 2, the relation between $w$ and $G_{M}$ is determined by

$$
w=\frac{\pi R_{0}{ }^{2}+\frac{m}{n} \pi\left(R_{\mathrm{w}}{ }^{2}-R_{0}{ }^{2}\right)}{A+\pi R_{0}{ }^{2}+\frac{m}{n} \pi\left(R_{\mathrm{w}}{ }^{2}-R_{0}{ }^{2}\right)}
$$

and

$$
G_{\mathrm{M}}=\frac{N}{3 n} G_{\mathrm{p}}^{\mathrm{res}}\left[\frac{n-m}{n}+\frac{m}{n} \frac{G_{\mathrm{p}}^{\mathrm{res}}}{G_{\mathrm{p}}^{\mathrm{s}}}\right]^{-1}
$$

for the serial type model, in explicit form

$$
\begin{aligned}
& G_{\mathrm{M}}(w)= \\
& \quad \frac{N}{3 n} G_{\mathrm{p}}^{\mathrm{res}}\left[1+\frac{1}{\pi\left(R_{\mathrm{w}}{ }^{2}-R_{0}{ }^{2}\right)}\left(A \frac{w}{1-w}-\pi R_{0}{ }^{2}\right)\left(\frac{G_{\mathrm{p}}^{\mathrm{res}}}{G_{\mathrm{p}}^{\mathrm{s}}}-1\right)\right]^{-1}
\end{aligned}
$$

If extended to higher dimensions, an approximate expression for the membrane conductance in the two-state model (2) is provided by $\mathrm{EMT}^{11}$ (cf. eq 34)

$$
\frac{m}{n} \frac{G_{\mathrm{p}}^{\mathrm{e}}-G_{\mathrm{p}}^{\mathrm{s}}}{G_{\mathrm{p}}^{\mathrm{s}}-(z / 2-1) G_{\mathrm{p}}^{\mathrm{e}}}+\left(1-\frac{m}{n}\right) \frac{G_{\mathrm{p}}^{\mathrm{e}}-G_{\mathrm{p}}^{\mathrm{res}}}{G_{\mathrm{p}}^{\mathrm{res}}-(z / 2-1) G_{\mathrm{p}}^{\mathrm{e}}}=0
$$

where $n$ now represents the total number of cylindrical segments in the network. Within both variants of the two-state model, given by eqs 55 and 57, $m$ varies in the range $0<m<n$. Equation 57 is an elementary cubic equation in $G_{\mathrm{p}}^{\mathrm{e}}$. It can be solved for $G_{\mathrm{p}}^{\mathrm{e}}$ as a function of $\mathrm{m} / \mathrm{n}$, finally determining a dependence $G_{\mathrm{p}}^{\mathrm{e}}(w)$. The resulting expression is, however, rather cumbersome. Therefore, we do not write it explicitly, but use it to plot the results. The solution $G_{\mathrm{p}}^{\mathrm{e}}(w)$ is shown in Figure 6 for the two limiting cases. It is proportional to $G_{\mathrm{M}}(w)$, as discussed in section IX.A. This figure demonstrates the effect 


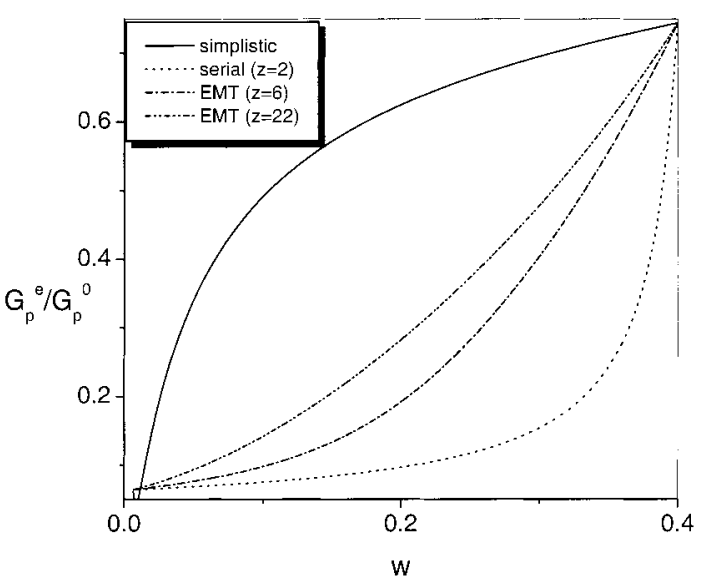

Figure 6. Effective conductance $G_{\mathrm{p}}^{\mathrm{e}}$ as a function of water volume fraction $w$ obtained within the two limiting cases of a $\delta$ function-like psd, corresponding to the simplistic model (eq 53) and the two-state model. The $G_{\mathrm{p}}(R)$ dependence depicted in Figure $3 \mathrm{c}$ is used as a basis. For the two-state model, conductances were calculated within the serial model variant (eq 56) as well as within the effective medium theory (EMT, eq 57) for two different coordination numbers $z$ (6 and 22).

of the porous structure on $G_{\mathrm{p}}^{\mathrm{e}}(w)$. The monodisperse psd (simplistic model), corresponding to a homogeneously evolving pore network upon water uptake, gives a superior conductance compared to the heterogeneous two-state model represented by the EMT or by a serial model. For the first, the conductance of a single pore carries over to the membrane conductance. For the latter, the network interconnectivity is essential; the worst performance is obtained within the serial model which does not account for pore space interconnectivity $(z=2)$. Greater conductances are calculated within EMT. The larger the coordination number $z$ is, i.e., the better the interconnectivity of the network is, the better the conductance is. The simplistic model overestimates the conductance, whereas the two-state model underestimates it. The real performance of polymer electrolyte membranes can be expected to lie within the boundaries set by these two bracketing results.

B. General Case. Model pore volume distributions $R^{2} g(R)$ $\left[=v_{\mathrm{w}} /\left(N L_{\mathrm{e}} \pi\right)\right]$, generated with eq 51 , are shown in panels a and b of Figure 7 at various $w$ values. Depending on the swelling parameter $t$, the distributions that evolve from the monodisperse initial configuration look rather different. $t=1.002$ gives a narrow, sharply peaked psd, which broadens only slightly with increasing water content. $t=1.1$ gives a broad psd, with a long tail to large radii that broadens substantially with increasing $w$ values. The first case closely resembles a monodisperse psd, whereas the latter mimics a polydisperse psd.

Functions $G_{\mathrm{p}}^{\mathrm{e}}(w)$, obtained by inserting the model psds of panels a and b of Figure 7 into eqs 31 and 32 (serial model) or eq 34 (EMT), are depicted in Figure 8. The results obtained with the narrow psd $(t=1.002$ in Figure $7 \mathrm{a})$ coincide precisely with those obtained within the simplistic model (solid curve in Figure 5). This means that, for this case, the single-pore relation $G_{\mathrm{p}}(R)$ carries over to the membrane conductance according to eq 53.

For the broad, polydisperse psd obtained with $t=1.1$, the serial model (dotted line in Figure 8) and EMT (O) give fairly different results for $G_{\mathrm{p}}^{\mathrm{e}}(w)$. The serial model, which neglects interconnectivity of pores, underestimates the conductance. For $t=1.1$, the relation $G_{\mathrm{p}}^{\mathrm{e}}(w)$ is, however, strongly determined by the single-pore property $G_{\mathrm{p}}(R)$, as can be seen by the comparison with the data calculated within the two-state model (eq 57) that are depicted in Figure 6.
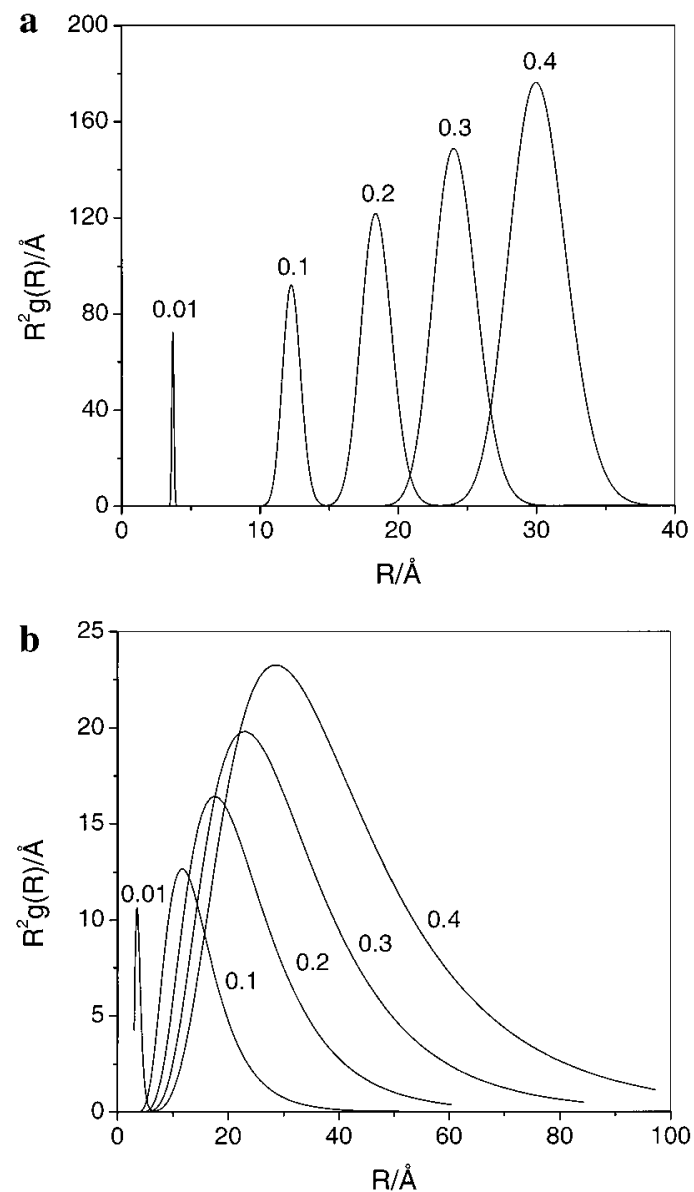

Figure 7. Pore volume distribution functions $R^{2} g(R)$ for two different values of the swelling parameter $t$, corresponding to (a) narrow distributions (1.002) and (b) broad distributions (1.1). The distributions are depicted for various water contents $w$, indicated as numbers at the curves.

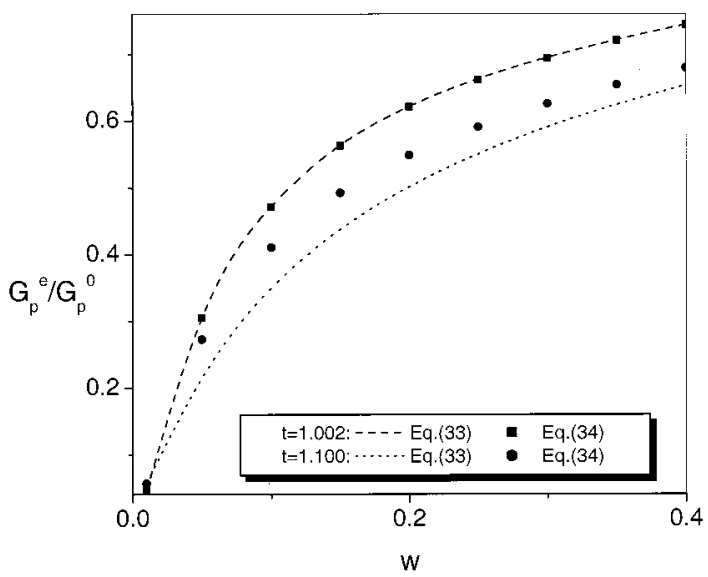

Figure 8. Conductance relations $G_{\mathrm{p}}^{\mathrm{e}}(w)$ calculated within the serial model (eq 32) and the effective medium theory (eq 34) for the two values of the swelling parameter $t$ and the associated pore volume distributions depicted in Figure 7.

\section{Discussion of Membrane Conductance}

Methods which allow incorporation of the single-pore property $G_{\mathrm{p}}(R)$ into contemporary models of the membrane porous structure were developed in section IX, and the corresponding results were presented in section $\mathrm{X}$. All the calculations are based on the assumption that the pores in the membrane have cylindrical cross sections. Furthermore, a regular swelling procedure in which a single relationship $G_{\mathrm{p}}(R)$ applies to all 
pores is presupposed. An initial monodisperse psd is fixed with all radii equal to the thickness $\delta$ of the surface water layer.

The integral relations, eqs $30-32$ and 34 , in section IX.A between psd $g(R)$, membrane water content $w$, and membrane conductance $G_{\mathrm{M}}$ provide the general framework. For specified $G_{\mathrm{p}}(R)$ and $g(R)$ values, these relations give $G_{\mathrm{M}}$ as a function of $w$. Two main variants have been studied. These are the serial model (cf. eq 32) and the random network model (cf. eq 34). The serial model reduces in the limiting case of a very narrow psd to the simplistic model, which decribes an entirely homogeneous system of equally swelling identical parallel tubes.

An appropriate model for a very heterogeneous membrane is, on the contrary, the two-state model. For the latter, a random network approach based on the effective medium approximation was studied in detail in ref 11. There, the two-state model provided results that were in excellent agreement with experimental data for Nafion if appropriate assumptions were made about the swelling procedure upon water uptake. The framework presented here is more universal. It includes various options for the single-pore property $G_{\mathrm{p}}(R)$ and for the psd $g(R)$, recovering also the two-state model as a limiting case.

To specify $g(R)$, an appropriate model of pore space evolution was presented in section IX.B. The continuous swelling procedure is split into elementary steps. At each step, the changes in $w$ and $G_{\mathrm{M}}$ can be calculated. In the continuum limit, it was shown that the procedure gives a log-normal psd typical of Nafion and its relatives.

The pore evolution model provides analytical solutions in the limit of small water contents for the serial approach (cf. section IX.D). A result that is highly relevant for polymer electrolyte membranes is the asymptotic behavior given by eq 45 . It predicts a linear dependence of $G_{\mathrm{M}}(w)$ for $w$ close to the residual water content $w^{\text {res }}$. The slope depends on the skewness parameter $t$ $(\geq 1)$ of pore space evolution. The largest slope is obtained with $t=1$.6. The closer $t$ is to 1, i.e., the narrower the psd, the smaller this slope is and the more important the quadratic term in eq 45 is.

With the help of the pore evolution model, different lognormal psds have been generated (see panels a and b of Figure 7 ), which allow calculation of $G_{\mathrm{M}}(w)$ for arbitrary $w$ values via eq 32 or 34 . With $t$ close to 1 , the resulting psd is very narrow, almost monodisperse. With increasing $w$ values, this psd broadens only slightly, i.e., the evolution of the pore structure is very homogeneous. In this case, the membrane conductance is well approximated by the simplistic model, as suggested by the comparison of the simplistic model results in Figure 6 and the result for the serial model with psd generated at $t=1.002$ in Figure 8. The conductance relation of the membrane is then predominantly determined by the single-pore properties, as given by eq 53 .

If $t$ deviates significantly from 1 , then a polydisperse psd is obtained which broadens substantially with increasing $w$ values (see Figure 7b). The evolving membrane structure becomes heterogeneous. The membrane conductance for this case is inferior compared to that of the homogeneous case.

In ref 11 , it was found that the two-state model with due account of swelling properties gave results that are in good agreement with experimental data for Nafion. ${ }^{25}$ In this paper, various alternative options have been studied and compared to each other, ranging from the simplistic model to the two-state model. The simplistic model gives the highest conductivity data. The data obtained within the effective medium approach using a narrow psd are very close to those of a simplistic model. These data, however, seem to overestimate the experimental data for Nafion. ${ }^{25}$

For the purpose of comparison of different model variants of pore space formation (simplistic, serial, and network), the results have been presented and discussed in terms of $G_{\mathrm{p}}^{\mathrm{e}}(w)$, normalized to $G_{\mathrm{p}}^{0}$. The overall membrane conductance $G_{\mathrm{M}}(w)$ is related to the effective single-segment conductance $G_{\mathrm{p}}^{\mathrm{e}}(w)$ by means of a proportionality factor $N / 3 n$ (cf. eq 32). To provide a numerical estimate of the specific membrane conductivity $\sigma_{\mathrm{M}}(w), N$ is replaced by the number density $v_{N}$ of tube entrances per unit surface area of the membrane (perpendicular to the direction of transport), and $n$ is replaced by the number density $v_{n}$ of segments in a single tube per unit $l_{\mathrm{p}}$ of apparent membrane thickness. $v_{n}$ is related to the tortuousity factor $\tau$, defined in Section IX, by

$$
\sqrt{\tau}=v_{n} l_{\mathrm{p}}
$$

and thus,

$$
\sigma_{\mathrm{M}}(w)=\frac{v_{N}}{3 v_{n}} G_{\mathrm{p}}^{\mathrm{e}}(w)=\frac{v_{N}}{3 \sqrt{\tau}} l_{\mathrm{p}} G_{\mathrm{p}}^{\mathrm{e}}(w)
$$

An estimate with $w=0.4$ at $\sqrt{\left\langle R^{2}\right\rangle}=30 \AA, \tau=3$, and $l_{\mathrm{p}}=50$ A gives

$$
\sigma_{\mathrm{M}}(w \approx 0.4)=1.8 \times 10^{5} \mathrm{~cm}^{-1} G_{\mathrm{p}}^{\mathrm{e}}(w=0.4)
$$

Using the previously obtained estimate $G_{\mathrm{p}}^{0}=5.5 \times 10^{-7} \mathrm{~S}$ and $G_{\mathrm{p}}^{\mathrm{e}}(w=0.4) / G_{\mathrm{p}}^{0}=0.75$, the overall estimate

$$
\sigma_{\mathrm{M}}(w=0.4) \approx 0.07 \mathrm{~S} \mathrm{~cm}^{-1}
$$

is obtained, demonstrating a good agreement with experimental data for Nafion $117 .^{25}$

\section{Conclusions}

This paper presents a comprehensive and systematic theoretical treatment of the proton conductance of polymer electrolyte membranes. Its construction is based on the assumption of a heterogeneous membrane structure of nonconducting hydrophobic polymer material and water-filled channels (pores) within which proton motion takes place. Under this assumption, we have developed a picture of proton transport in a channel, and proceeded to incorporate this picture into a statistical theory of a multichannel structure. The statistical theory has, as constraints, certain "sum rules" imposed by the observed pore size distribution and the laws of swelling. This, together with the detailed analysis of plausible events in one pore, changes the character of the theory from a purely descriptive one; it acquires certain predictive power and a power of rationalizing the main factors which influence the proton conductivity as a function of water content.

In a single pore, we have distinguished surface and bulk mechanisms of proton transport. The surface mechanism is characterized by a higher activation energy and, thereby, lower mobility, but also by a higher concentration of charge carriers, which are accumulated near the negatively charged pore surface. The balance between surface and bulk contribution is determined by the surface density of $\mathrm{SO}_{3}{ }^{-}$groups and the pore size. A higher density of anionic groups gives rise to high surface mobility, considerably improving proton transport even for a relatively dry state. Larger pore radii open the possibilities in which the protons can reside and move through the bulk, where they have higher mobility. Thus, the overall conductance of the 
pore increases with its radius, until it reaches a saturation, which happens at $R \gtrsim 30 \AA$. Here the effect of the increase in the volume of the highly mobile bulklike region is compensated by the lowering of the average proton density in the pore. The fact that there is a saturation, i.e., there is no under- or overcompensation is nontrivial, and the theory shows why this is so.

The pore structure effects were treated by two alternative models: the serial model (the particular case of which is a simplistic model of straight parallel channels) and the random network model. The true structure of the membrane lies somewhere "in between". Both models can be matched to pore size distributions of any width, and were parametrized to reproduce experimentally obtained distributions. For broad pore size distributions, however, the serial model yields a performance which is too low, since even a small amount of narrow fragments will dramatically diminish the overall performance of the channels. Such behavior is also reflected in the random network model, in which the conductance of the membrane with a monodisperse pore size distribution is higher than that of a broad distribution with same mean pore radius and all other factors equal.

The theory establishes a closed form relationship between the conductance, water uptake, and porous structure. The relationship is purely analytical in important limiting cases or has a form of parametric solution. Its qualitative predictions are in line with available experimental data. Moreover, specifying a reasonable set of parameters for a saturated Nafion membrane, we obtained a numerical estimate of the specific membrane conductivity which demonstrates excellent agreement with experimental values.

To obtain this closed form solution, however, we had to make a number of simplifying assumptions, the relaxation of which could add interesting new capabilities to the theory. Allowing for agglomeration or correlation of sizes in swelling or imposing sharp constraints on the minimum and maximum pore sizes could make the description richer, at the cost of mathematical transparency. For these goals, computer random network simulations would be more appropriate.

The future task will be to integrate such models with direct molecular dynamics simulations of elementary proton transfer events within chemically specific models of the membrane structure; these simulations are currently underway. The theory presented here offers a promising framework for the extension of such simulation results, which are limited by computational complexity to studies of nanoscale membrane structures, from the atomic to the engineering scale.

Acknowledgment. We are thankful to V. I. Gordelyi and A. V. Neimark for useful discussions. This work was performed as a part of a project "Membranes and Membrane Electrode Assemblies for Direct Methanol Fuel Cells", supported by the German government under BMWi Grant 0327086 A. Furthermore, it was supported by the Danish Technical Science Foundation. S.W. thanks the Alexander von Humboldt Foundation for their support. A.A.K., A.M.K., and J.U. acknowledge the support of INTAS (project \#99-1093).

\section{Appendix A: Transfer between Sulfonate Groups on the Surface of the Pore}

When the distance between neighboring $\mathrm{SO}_{3}{ }^{-}$positions $\mathrm{A}$ and $\mathrm{C}$ is not too large ( $\$ 7 \AA$ ), we my assume that there is one intermediate symmetric position $\mathrm{B}$ between them accepting the proton at the first step on its way from $\mathrm{A}$ to $\mathrm{C}$. The rate constant or the transition probability of this two-step process is

$$
k_{\mathrm{AC}}=\frac{k_{\mathrm{AB}} k_{\mathrm{BC}}}{k_{\mathrm{BA}}+k_{\mathrm{BC}}}=\frac{1}{2} k_{\mathrm{AB}}
$$

since in the symmetric case $k_{\mathrm{BA}}=k_{\mathrm{BC}}$.

The expression for the transition probability is then given by eq 4. However, when the distance between groups A and D increases, we may expect that after some distance the proton will need two intermediate sites (B and $\mathrm{C}$ ) for the transition from A to D. Thus, the change in the transition probability with the increase in the pore size upon water uptake will be due to the change in the transfer mechanism rather than to the change in the reorganization energy with the increase in the transfer distance.

In view of the symmetry, one may assume that the electron energy levels of sites B and C are equal although different from (higher than) those of groups A and D. The transition probability or the rate constant for the transition from $\mathrm{A}$ to $\mathrm{D}$ can be calculated using an ordinary steady state approximation for the concentrations of the intermediates. The solution has the form

$$
\frac{1}{k_{\mathrm{AD}}}=\frac{1}{k_{\mathrm{AB}}}\left(2+\frac{k_{\mathrm{BA}}}{k_{\mathrm{BC}}}\right)
$$

and since the last term in the brackets is much larger than unity, we finally obtain

$$
k_{\mathrm{AD}}=k_{\mathrm{BC}} \frac{k_{\mathrm{AB}}}{k_{\mathrm{BA}}}
$$

Thus, the activation energy here is different from that in the former case; it is determined by the sum of the activation free energy of the transition from site $\mathrm{A}$ to $\mathrm{B}$ and the activation free energy for the transition from site $\mathrm{B}$ to $\mathrm{C}$ minus the activation free energy of the reverse B to A process. The latter cannot be larger than that of the A to B transfer, and the overall effective activation energy can only increase due to the intermediate step.

At even larger distances between $\mathrm{SO}_{3}{ }^{-}$groups, three intermediate sites (water molecules), B-D, may be involved in the transition from donor groups A to acceptor group E. In view of symmetry, we may expect the energies of sites B and D to be equal to each other (although different from those of the donor and acceptor groups), and the energy of site $\mathrm{C}$ may be different (somewhat higher) from all others. The rate constant (transition probability) is then

$\frac{1}{k_{\mathrm{AE}}}=\frac{1}{k_{\mathrm{AB}}}+\frac{k_{\mathrm{BA}}}{k_{\mathrm{AB}}} \frac{1}{k_{\mathrm{BC}}}+\frac{k_{\mathrm{CB}}}{k_{\mathrm{BC}}} \frac{k_{\mathrm{BA}}}{k_{\mathrm{AB}}} \frac{1}{k_{\mathrm{CD}}}+\frac{k_{\mathrm{DC}}}{k_{\mathrm{CD}}} \frac{k_{\mathrm{CB}}}{k_{\mathrm{BC}}} \frac{k_{\mathrm{BA}}}{k_{\mathrm{AB}}} \frac{1}{k_{\mathrm{DE}}}$

The first and last terms on the right-hand side of this equation are most probably small, and we have approximately

$$
\begin{aligned}
\frac{1}{k_{\mathrm{AE}}} \approx & \frac{1}{k_{\mathrm{BC}}^{0}} \exp \left(\frac{\Delta G_{\mathrm{AB}}+E_{\mathrm{r}}^{\mathrm{BC}} / 4+\Delta G_{\mathrm{BC}} / 2}{k_{\mathrm{B}} T}\right)+ \\
& \frac{1}{k_{\mathrm{CD}}^{0}} \exp \left(\frac{\Delta G_{\mathrm{AB}}+\Delta G_{\mathrm{BC}}+E_{\mathrm{r}}^{\mathrm{BC}} / 4-\Delta G_{\mathrm{BC}} / 2}{k_{\mathrm{B}} T}\right)= \\
& \exp \left(\frac{\Delta G_{\mathrm{AB}}+E_{\mathrm{r}}^{\mathrm{BC}} / 4+\Delta G_{\mathrm{BC}} / 2}{k_{\mathrm{B}} T}\right)\left(\frac{1}{k_{\mathrm{BC}}^{0}}+\frac{1}{k_{\mathrm{CD}}^{0}}\right)
\end{aligned}
$$

where $k_{\mathrm{BC}}^{0}$ and $k_{\mathrm{CD}}^{0}$ are pre-exponential factors and it was taken into account that $E_{\mathrm{r}}^{\mathrm{BC}}=E_{\mathrm{r}}^{\mathrm{CD}}$ due to symmetry. Equation $\mathrm{A} 5$ demonstrates the overall enhancement of the effective activation energy of the multistep transition. 
Thus, when the pore size varies with wetting, at some intergroup distances the mechanism of the proton transfer may change abruptly due to the change in the structure of the water near the surface groups, but the tendency of the apparent activation energy increase remains valid.

\section{Abbreviations}

EMT effective medium theory

EW equivalent weight (number of grams of dry polymer per mole of anionic groups)

psd pore size distribution

PT proton transfer description

\section{List of Symbols}

Roman letters

$a_{\mathrm{b}}$

$a_{\mathrm{s}}$

A

$A_{i}$

$e$

$E_{\mathrm{r}}$

$E_{\mathrm{b}}$

$E_{\mathrm{s}}$

$\Delta G$

$G_{\mathrm{p}}^{0}$

$G_{\mathrm{p}}$

$G_{\mathrm{p}}^{\mathrm{e}}$

$G_{\mathrm{p}}^{\mathrm{res}}$

$G_{\mathrm{p}}^{\mathrm{s}}$

$G_{\mathrm{M}}(w)$

$g(R)$

effective distance of the net charge transfer (bulk) effective distance of the net charge transfer (surface) effective "area", a parameter of the dry membrane cross sections of elementary tubes $(i=1, \ldots, n)$ elementary charge $\left(1.602 \times 10^{-19} \mathrm{C}\right)$

reorganization Gibbs free energy reorganization Gibbs free energy (bulk) reorganization Gibbs free energy (surface) driving force term in surface activation energy reference single-pore condcutance, defined in eq 22 conductance of an individual cylindrical segment effective conductance of a representative individual cylindrical segment in EMT

residual conductance of a dry individual cylindrical segment

saturation conductance of a water-swollen individual cylindrical segment

effective conductance of the membrane

"pore size distribution" function, abbreviated as psd product of geometry factor and coordination number in expressions for proton mobility (surface, bulk, and radius-dependent values, respectively)

$k_{\mathrm{B}} \quad$ Boltzmann constant $\left(1.38 \times 10^{-23} \mathrm{~J} \mathrm{~K}^{-1}\right)$

$k_{\mathrm{s}}, k_{\mathrm{b}}$, and $k(r)$ geometrical factor in expressions for proton mobility (surface, bulk, and radius-dependent values, respectively)

$l$

$L_{\text {B }} \quad$ Bjerrum length

$L_{\mathrm{p}}$

$l_{\mathrm{p}}$

$M_{\mathrm{w}}$ and $V_{\mathrm{w}}$

$M_{\mathrm{p}}$ and $V_{\mathrm{p}}$

$M$

$m$

$N$

$n$

$P(m)$ membrane)

length of an elementary cylindrical pore segment mass and volume of the water fraction, respectively

mass and volume of a dry polymer material, respectively

total number of elementary expansion/shrinking procedures

number of elementary expansion/shrinking procedures for a single segment

total number of parallel cylindrical tubes

number of elementary cylindrical segments into which the randomly constricted, sample-spanning tube is subdivided

probability for any particular segment $i$ to shrink exactly $m$

radial coordinate in an individual pore

pore radius
$R_{0}$

$R$ tube

$t$

$T$

$T^{0}$

$v_{\mathrm{w}}(R)$

$w$

$W_{\mathrm{b}}$

$W_{\mathrm{s}}$

$z$

List of Symbols

Greek letters

$\Delta$

$\delta$

$\epsilon_{\mathrm{b}}$ and $\epsilon_{\mathrm{s}}$

$\epsilon_{\mathrm{b}}$

$\epsilon_{\mathrm{s}}$

$\theta$

$\Theta$

$\Lambda$

$\mu_{\mathrm{b}}$

$\mu_{\mathrm{s}}$

$\mu(r)$

$v_{\mathrm{s}}, v_{\mathrm{b}}$, and $v(r)$

$v_{N}$

$v_{n}$

$\rho_{\mathrm{s}}$

$\rho_{0}$

$\rho(r)$

$\rho_{\mathrm{p}}$

$\rho_{\mathrm{w}}$

$\Sigma$

pore radius of an initial monodisperse distribution in a dry membrane

resistance of the serial array of conductances in one tube

expansion/shrinking parameter applied to an individual cylindrical segment

temperature

reference temperature $(373 \mathrm{~K})$

differential water volume distribution

membrane water content in terms of volume $\left(w_{\mathrm{V}}\right)$ or mass fraction $\left(w_{\mathrm{M}}\right)$

transition probability per unit time of bulk mechanism transition probability per unit time of surface mechanism

coordination number of pores, a phenomenological parameter in the effective medium approximation description

$R$-dependent variable $\left[\Delta=(1-\delta / R)^{2}\right]$

thickness of a surface water layer $(\approx 3 \AA)$

dielectric constants of water in bulk and in a surface layer, respectively

energy needed for the configurational preorganization of the solvated proton transfer complex (bulk)

energy needed for the configurational preorganization of the solvated proton transfer complex (surface)

thermal energy $\left(=k_{\mathrm{B}} T\right)$

Heaviside-step function $[\Theta(x)=1$ for $x>0$ and zero otherwise]

total number of $\mathrm{SO}_{3}{ }^{-}$groups (and protons) in the pore proton mobility in the bulk

proton mobility at the surface

mobility for a cylindrical pore model

coordination number in expressions for proton mobility (surface, bulk, and radius-dependent values, respectively)

number density of tubes per unit membrane surface number density of segments per unit of apparent membrane thickness

proton concentration in the surface layer

proton concentration at the center of the pore

proton distribution in the bulk of the pore

volume density of a dry polymer material

volume density of water

surface charge density of sulfonic acid headgroups on a pore wall

tortuousity factor

electrostatic potential in the pore

effective frequency of all the environmental modes (bulk)

effective frequency of all the environmental modes (surface)

\section{References and Notes}

(1) Doyle, M.; Choi, S. K.; Proulx, G. J. Electrochem. Soc. 2000, 147,

(2) Kerres, J.; Cui, W.; Disson, R.; Neubrand, W. J. Membr. Sci. 1998, 139, 211.

(3) Brack, H. P.; Büchi, F. N.; Huslage, J.; Rota, M.; Scherer, G. G. ACS Symp. Ser. 1999, 744, 174.

(4) Lehtinen, T.; Sundholm, G.; Holmberg, S.; Sundholm, F.; Björnbom, P.; Bursell, M. Electrochim. Acta 1998, 43, 1881. 
(5) Kreuer, K. D.; Fuchs, A.; Ise, M.; Spaeth, M.; Maier, J. Electrochim. Acta 1998, 43, 1281.

(6) Paddison, S. J.; Paul, R.; Zawodzinski, Th. A. J. Electrochem. Soc. 2000, 147,617 .

(7) Kordesch, K.; Simader, G. Fuel Cells and Their Applications; VCH: Weinheim, Germany, 1996.

(8) Narayanan, S. R.; Kindler, A.; Jeffries-Nakamura, B.; Chun, W.; Frank, H.; Smart, M.; Valdez, T. I.; Surampudi, S.; Halpert, G.; Cosek, J.; Cropley, C. In Proceedings of the 11th Annual Battery Conference on Application and Advances; IEEE: Long Beach, CA, 1996; p 113.

(9) Eikerling, M.; Kharkats, Yu. I.; Kornyshev, A. A.; Volfkovich, Yu. M. J. Electrochem. Soc. 1998, 145, 2684.

(10) Kornyshev, A. A.; Walbran, S. J. Chem. Phys., in press.

(11) Eikerling, M.; Kornyshev, A. A.; Stimming, U. J. Phys. Chem. B 1997, 101, 10807.

(12) Kuznetsov, A. M.; Ulstrup, J. Electron Transfer in Chemistry and Biology; Wiley Series in Theoretical Chemistry; John Wiley \& Sons Ltd.: New York, 1998.

(13) Sahimi, M. Flow and Transport in Porous Media and Fractured Rock: From Classical Methods to Modern Approaches; VCH: Weinheim, Germany, 1995

(14) Wong, P.; Koplik, J.; Tomanic, J. P. Phys. Rev. B 1984, 30, 6606.

(15) Agmon, N. J. Phys. Chem. 1996, 100, 1072.

(16) Kreuer, K. D. Proton Conductors: Materials and Applications, Chemistry of Materials; 1996; Vol. 8, pp 610-641.

(17) Marx, D.; Tuckerman, M. E.; Hutter, J.; Parinello, M. Nature 1999 397, 601 .

(18) Schmidt, U. W.; Voth, G. A. J. Chem. Phys. 1999, 111, 9361.

(19) Vuilleumier, R.; Borgis, D. J. Chem. Phys. 1999, 111, 4251.

(20) Zundel, G.; Metzger, H. Energiebänder der tunnelnden ÜberschussProtonen in flüssigen Säuren. Eine IR-spektroskopische Untersuchung der Natur der Gruppierungen $\mathrm{H}_{5} \mathrm{O}_{2}{ }^{+}$. Z. Phys. Chem. 1968, 58, 225.

(21) Wicke, E.; Eigen, M.; Ackermann, Th. Über den Zustand des Protons (Hydroniumions) in wässriger Lösung. Z. Phys. Chem. 1954, 1 , 340.

(22) Ennari, J. Ph.D. Dissertation, University of Helsinki, Helsinki, 2000.

(23) Atkins, P. W. Physical Chemistry, 6th ed.; Oxford University Press: Oxford, U.K., 1998; Chapter 24.8, 741.

(24) Sadeghi, R. R.; Cheng, H.-P. J. Phys. Chem. B 1999, 103, 2086.

(25) Zawodzinski, T. A.; Derouin, C.; Radzinski, S.; Sherman, R. J.; Smith, V. T.; Springer, T. E.; Gottesfeld, S. J. Electrochem. Soc. 1993 140, 1041.

(26) Kreuer, K. D.; Dippel, T.; Meyer, W.; Maier, J. Mater. Res. Soc. Symp. Proc. 1993, 293, 273.

(27) Gierke, T. D.; Munn, G. E.; Wilson, F. C. J. Polym. Sci. Polym. Phys. Ed. 1981, 19, 1687.

(28) Kornyshev, A. A.; Kuznetsov, A. M.; Ulstrup, J.; Stimming, U. J. Phys. Chem. B 1997, 101, 5917.
(29) Myers, H. P. Introductory Solid State Physics; Taylor and Francis: London, 1997.

(30) Litt, M. H. Polym. Prepr. 1997, 38, 80

(31) Mosdale, R.; Gebel, G.; Pineri, M. J. Membr. Sci. 1996, 118, 269.

(32) Kornyshev, A. A. In The Chemical Physics of Solvation, Part A. Theory of Solvation; Dogonadze, R. R., Kálmán, E., Kornyshev, A. A., Ulstrup, J., Eds.; Elsevier: Amsterdam, 1985; p 77.

(33) Vorotyntsev, M. A.; Kornyshev, A. A. Electrostatics of Media with Spatial Dispersion; Nauka: Moscow, 1993.

(34) Kornyshev, A. A.; Tsitsuashvili, G. I.; Yaroschuk, A. E. Elektrokhimiya 1989, 25, 1027.

(35) Kornyshev, A. A.; Tsitsuashvili, G. I.; Yaroschuk, A. E. Elektrokhimiya 1989, 25, 1037.

(36) Eikerling, M.; Kornyshev, A. A. J. Electroanal. Chem. 2001, 502 ,

(37) Cappadonia, M.; Erning, J. W.; Niaki, S. M.; Stimming, U. Solid State Ionics 1995, 77, 65 .

(38) Cappadonia, M.; Kornyshev, A. A.; Krause, S.; Kuznetsov, A. M.; Stimming, U. J. Chem. Phys. 1994, 101, 7672.

(39) Dullien, F. A. L. Porous Media, Fluid Transport and Porous Structure; Academic Press: New York, 1979; Chapter 4.

(40) Scheidegger, A. E. The Physics of Flow Through Porous Media, 3rd ed.; University of Toronto Press: Toronto, 1974.

(41) Archie, G. E. Trans. AJME 1942, 146, 54.

(42) Gordelyi, V. Unpublished data.

(43) Schlick, S., Ed. Ionomers: characterization, theory, and applications; CRC Press: Boca Raton, FL, 1995.

(44) Chizmadzhev, Yu. A.; Markin, V. S.; Tarasevich, M. R.; Chirkov, Yu. G. Macrokinetics of Processes in Porous Media (Fuel Cells), Chapter 6: Transfer Processes in Porous Media; Nauka: Moscow, 1971 (in Russian).

(45) Kirkpatrick, S. Percolation and Conduction. Rev. Mod. Phys. 1973, 45 (4), 574.

(46) Kheifets, L. I.; Neimark, A. V. Theor. Found. Chem. Eng. 1983, $17,158$.

(47) Koplik, J. J. Phys. C: Solid State Phys. 1981, 14, 4821.

(48) Bernasconi, J.; Wiesmann, H. J. Phys. Rev. B 1976, 13, 1131.

(49) Divisek, J.; Eikerling, M.; Mazin, V.; Schmitz, H.; Stimming, U.; Volfkovich, Yu. M. J. Electrochem. Soc. 1998, 145, 2677.

(50) Eikerling, M. Theoretical Modelling of the Electrophysical Properties, Structure, and Functioning of Low-Temperature Ion Exchange Membranes. Ph.D. Dissertation, TU München, München, Germany, 1999.

(51) Baltus, R. E. J. Membr. Sci. 1997, 123, 165.

(52) Verbrugge, M. W.; Hill, R. F. J. Electrochem. Soc. 1990, 137, 886.

(53) Stauffer, D.; Aharony, A. Introduction to Percolation Theory, 2nd ed.; Taylor and Francis: London, 1994 (Figure 13.1). 